\title{
Steric effects in the dynamics of electrolytes at large applied voltages: I. Double-layer charging
}

\author{
Mustafa Sabri Kilic and Martin Z. Bazant \\ Department of Mathematics, Massachusetts Institute of Technology, Cambridge, MA 02139,USA.
}

\begin{abstract}
Armand Ajdari
Labortoire de Physico-Chimie Theorique, UMR ESPCI-CNRS 7083, 10 rue Vauquelin, F-75005 Paris, France.
\end{abstract} (Date textdate; Received textdate; Revised textdate; Accepted textdate; Published textdate)

\begin{abstract}
The classical Poisson-Boltzmann (PB) theory of electrolytes assumes a dilute solution of point charges with mean-field electrostatic forces. Even for very dilute solutions, however, it predicts absurdly large ion concentrations (exceeding close packing) for surface potentials of only a few tenths of a volt, which are often exceeded, e.g. in microfluidic pumps and electrochemical sensors. Since the 1950s, several modifications of the PB equation have been proposed to account for the finite size of ions in equilibrium, but in this two-part series, we consider steric effects on diffuse charge dynamics (in the absence of electro-osmotic flow). In this first part, we review the literature and analyze two simple models for the charging of a thin double layer, which must form a condensed layer of close-packed ions near the surface at high voltage. A surprising prediction is that the differential capacitance typically varies non-monotonically with the applied voltage, and thus so does the response time of an electrolytic system. In PB theory, the capacitance blows up exponentially with voltage, but steric effects actually cause it to decrease above a threshold voltage where ions become crowded near the surface. Other nonlinear effects in PB theory are also strongly suppressed by steric effects: The net salt adsorption by the double layers in response to the applied voltage is greatly reduced, and so is the tangential "surface conduction" in the diffuse layer, to the point that it can often be neglected compared to bulk conduction (small Dukhin number).
\end{abstract}

\section{INTRODUCTION}

In this two-part series, we develop a simple analytical theory for the dynamics of electrolytes, taking into account steric effects of finite ion size. Motivated by recent experiments in microfluidics, microbatteries, and electrochemical sensors, our motivation is to describe the response of an electrolyte to an applied voltage of several volts, which is large enough to cause crowding of ions near a surface, even if the bulk solution is very dilute and in the absence of surface reactions. The ions thus lose their classical Poisson-Boltzmann distribution, which has major implications for their dynamics.

As a guide to the reader, we summarize the main results. The present "Part I" begins in this section with a historical review of dilute solution theory, its limitations at large voltages, and attempts to account for steric hindrance, specific interactions, and many-body electrostatics. As a first approximation, we focus only on steric effects, and analyze the dynamical response of a thin diffuse layer to a large applied voltage, using two simple continuum models (section III). The key results, common to both steric models, are: (i) the diffuse layer's differential capacitance is bounded and decreases at large voltage (section III), and (ii) it cannot easily engulf enough ions to perturb the bulk concentration or to conduct significant tangential currents (section IV). These predictions are completely opposite to those of dilute solution theory (based on the Gouy-Chapman model of the diffuse layer). In the companion paper "Part II", we propose general, time-dependent equations with steric constraints and revisit the parallel-plate charging problem in more detail.

\section{A. Dilute solution theory}

For the past century, dilute solution theory has provided the standard model of electro-diffusion of ions 59 , 70, 81] and electrokinetic phenomena [47, 60]. The fundamental assumption is that the chemical potential of a point-like ion $i$ in a dilute solution has the simple form,

$$
\mu_{i}=k T \ln c_{i}+z_{i} e \psi
$$

where $z_{i} e$ is the charge, $c_{i}$ the concentration, and $\psi$ the electrostatic potential, determined in a mean-field approximation by Poisson's equation,

$$
-\nabla \cdot(\varepsilon \nabla \psi)=\rho=\sum_{i} z_{i} e c_{i}
$$

typically with a constant permittivity $\varepsilon$. The form (1) is sometimes called the "ideal" component of the chemical potential [37], to which various "excess" components at finite concentration can be added (see below).

In many situations, it is assumed that the ions are in quasi-thermal equilibrium with a Boltzmann distribution,

$$
c_{i}=c_{i}^{0} e^{-z_{i} e \psi / k T}
$$

with a reference concentration $c_{i}^{0}$, in which case Equation (2) reduces to the Poisson-Boltzmann equation (PB). For example, to analyze a thin double layer, it is natural to choose $c_{i}^{0}$ to be the concentration of species $i$ in the nearby neutral bulk solution. In most situations, the $\mathrm{PB}$ equation is hard enough to solve that the the DebyeHückel linearization for small potentials, $|\psi| \ll \psi_{T}$, is 
required for analytical progress, where $\psi_{T}=k T / z_{i} e$ is the thermal voltage $(\approx 25 \mathrm{mV}$ for monovalent ions at room temperature).

The well known exception, which admits a simple solution, is the case of a symmetric binary $z: z$ electrolyte in a 1D geometry, where the PB equation in the form

$$
\varepsilon \frac{\partial^{2} \psi}{\partial x^{2}}=2 z e c_{0} \sinh \left(\frac{z e \psi}{k T}\right)
$$

was solved analytically by Gouy [41] and Chapman 22] (GC) for a semi-infinite electrolyte of bulk concentration $c_{0}=c_{+}^{0}=c_{-}^{0}$ near a flat charged surface [47, 59, 70]. (It is less well known that Gouy [41] also solved the case of an asymmetric $2 z: z$ electrolyte.) Although this solution may seem quite special, it describes the most common situation where diffuse charge is confined to a thin capacitor-like "double layer" near a solid surface. The width of the diffuse layer is the Debye [26] (or, more properly, Gouy [41]) screening length,

$$
\lambda_{D}=\sqrt{\frac{\varepsilon k T}{2 z^{2} e^{2} c_{0}}}
$$

as can be seen by scaling (44) with $\tilde{x}=x / \lambda_{D}$ and $\tilde{\psi}=\psi / \psi_{T}$ to obtain the dimensionless form, $\tilde{\psi}^{\prime \prime}=\sinh \tilde{\psi}$. The screening length $\left(\lambda_{D} \approx 1-100 \mathrm{~nm}\right.$ in aqueous electrolytes) is typically much smaller than any geometrical length, so bulk solution remains quasi-neutral with diffuse charge confined to thin, locally flat, quasiequilibrium double layers.

Due to its analytical simplicity and wide applicability, the Gouy-Chapman solution has become the standard model for highly charged double layers. It is the basis for the classical theory of tangential "surface conduction" through the diffuse layer [10, 11, 27, 30], as well as the recently noted phenomenon of salt "adsorption" (or "uptake") from the neutral bulk by the diffuse layer in response to a large applied voltage [6, 24]. Such predictions of the GC model have major implications for electrokinetic phenomena of the first kind, such as electro-osmosis, electrophoresis, streaming potential, and diffusiophoresis, at large surface potentials [47, 60].

Dilute solution theory has also been used in nearly every model of diffuse charge during the passage of current. Near equilibrium, the flux density of ion $i$ is proportional to the gradient of its chemical potential (1),

$$
F_{i}=-b_{i} c_{i} \nabla \mu_{i}=-D_{i}\left(\nabla c_{i}+\frac{z_{i} e}{k T} c_{i} \nabla \psi\right)
$$

where Einstein's relation, $D_{i}=b_{i} / k T$, relates the ion's mobility $b_{i}$ to its diffusivity $D_{i}$. For a system in quasisteady state, $\nabla \cdot F_{i}=0$, the nonzero current, $J=$ $\sum_{i} z_{i} e F_{i}$, only slightly perturbs the Boltzmann distribution of the ions. As a result, the GC solution is also widely used to describe diffuse-layer effects on electrode reaction kinetics [4, 70] (the Frumkin correction [34]), up to Nernst's limiting current, where the bulk concentration of an electro-active species vanishes. At larger "superlimiting" currents, the PB equation loses validity, but dilute-solution theory is still used to describe diffuse charge, which loses its Boltzmann distribution [91] and extends into the bulk as "space charge" 82], while retaining an inner boundary layer at the screening length [23]. The dilute-solution space-charge model is the basis for theories of electrokinetic phenomena of the second kind, such as super-fast electrophoresis [29] and hydrodynamic instability at a limiting current 83].

Dilute solution theory has also been used to describe the dynamics of electrolytes, subject to time-dependent applied voltages. The classical description comes from the Poisson-Nernst-Planck equations (PNP), which consist of Eq. (2) and mass conservation laws for the ions,

$$
\frac{\partial c_{i}}{\partial t}=-\nabla \cdot F_{i}=D_{i}\left[\nabla^{2} c_{i}+\frac{z_{i} e}{k T} \nabla \cdot\left(c_{i} \nabla \psi\right)\right]
$$

where $D_{i}=$ constant is normally assumed. Again, general solutions are only possible upon linearization, where the potential satsifies the Debye-Falkenhagen equation [26]. In the nonlinear regime, for thin double layers, the GC solution again holds since the diffusecharge remains in quasi-equilibrium, and the diffuse-layer acts like a voltage-dependent differential capacitance in series with a bulk resistor [62]. Such equivalent circuit models can be derived systematically from the PNP equations by aymptotic analysis, which also reveals corrections at large voltages, due to bulk diffusion in response to salt adsorption [6] and surface conduction 24] at large applied voltages. Such nonlinear diffuse-layer effects could be important in intepretting impedance spectra in electrochemical sensing [36, 63], or in understanding high-rate thin-film rechargeable batteries [28, 69, 95, 96].

Another current motivation to study nonlinear diffusecharge dynamics comes from "induced-charge" electrokinetic phenomena [5]. The preceding models of doublelayer relaxation have been used extensively in theories of AC pumping of liquids over electrode arrays [1, 7, 40, 78, 79? ], induced-charge electro-osmotic flows around metallic colloids [35, 66] and microstructures [7, 57, 92], and dielectrophoresis 87, 89, 93 and induced-charge electrophoresis [5, 25, 93, 98] of polarizable particles in electrolytes, although a nonlinear analysis based on the PNP equations has not yet been attempted for any of these situations. This may be a good thing, however, since we will show that dilute solution theory generally breaks down in the regime of experimental interest, where the applied voltage is much larger than the thermal voltage, $V>\psi_{T}$.

\section{B. Validity of the nonlinear model for dilute solutions}

Dilute solution theory provides a natural starting point to understand nonlinear effects in electrolytes, and the GC model is used in all of the examples above to model the diffuse layer at large applied potentials. In spite of its 
mathematical appeal, however, nonlinear dilute solution theory has limited applicability, due to the exponential sensitivity of the counter-ion concentration to voltage. For example, in the typical case of quasi-equilibrium, the Boltzmann factor (3) brings nonlinearity to the PB equation (4) when $\psi>\psi_{T}$, but this dependence is so strong that the surface potential cannot get much larger without invalidating the assumption of a dilute solution.

To see this, note that there must be a maximum concentration, $c_{\max }=a^{-3}$, of counterions packed with typical spacing $a$ near a highly charged surface. This effective ion size is clearly no smaller than the ionic radius (typically $\approx 1 \AA$ ), although it could be considerably larger (several $\mathrm{nm}$ ), taking into account hydration effects and ion-ion correlations, especially in a large electric field. For the sake of argument, consider a very dilute bulk solution, $c_{0}=10^{-5} \mathrm{M}$, with $a=3 \AA$ and $z=1$. The nonlinear regime begins at a diffuse-layer potential $\Psi_{D}$ $\approx k T / z e=25 \mathrm{mV}$, but the steric limit is reached in dilute solution theory at $\Psi_{c} \approx 13 k T / z e=330 \mathrm{mV}$, where

$$
\Psi_{c}=-\frac{k T}{z e} \ln \left(a^{3} c_{0}\right)=\frac{k T}{z e} \ln \left(\frac{c_{\max }}{c_{0}}\right) .
$$

Since the solution ceases to be "dilute" well below the steric limit, there is only a narrow window of surface potentials $(\approx 25-200 \mathrm{mV})$, sometimes called "weakly nonlinear regime" [6], where nonlinearity arises and the fundamental assumption (11) remains valid.

Unfortunately, the most interesting predictions of the non-linear PB theory tend to be in the "strongly nonlinear regime", where the dilute approximation fails dramatically. For example, the Dukhin number, which controls the relative importance of tangential conductivity in a thin diffuse layer, $\sigma_{s}$, compared to bulk conductivity, $\sigma$, at a geometrical scale $L$, has the following form [10, 11, 27],

$$
\mathrm{Du}=\frac{\sigma_{s}}{\sigma L}=4 \frac{\lambda_{D}}{L} \sinh ^{2}\left(\frac{z e \Psi_{D}}{4 k T}\right),
$$

assuming the Gouy-Chapman model. This is also the dimensionless ratio, $\Gamma_{s} / c_{0} L$, of the excess surface concentration of ions, $\Gamma_{s}$, relative to the bulk concentration, so it also governs the (positive) adsorption of neutral salt from the bulk in response to an applied voltage [6, 61]. The general derivation of Eq. (9) assumes a thin double layer [24], but in that case $\left(\lambda_{D} \ll L\right)$ a large Dukhin number corresponds to situations where the steric constraint is significantly violated, $\Psi_{D}>\Psi_{c}$, rendering Equation (9) inappropriate. Similar concerns apply to other nonlinear effects in Gouy-Chapman theory, such as the rapid increase of the differential capacitance (defined below) with surface potential,

$$
C_{D}=\frac{\varepsilon}{\lambda_{D}} \cosh \left(\frac{z e \Psi_{D}}{2 k T}\right)
$$

which would have important implications for electrochemical relaxation around conductors [6, 24, 88] and for AC electro-osmosis [3, 58].

\section{Beyond the Poisson-Boltzmann picture}

We are certainly not the first to recognize the limitations of dilute solution theory. Historically, concerns about the unbounded capacitance of a thin diffuse layer in the GC solution (10) first motivated Stern 94 to hypothesize that there must also be compact layer of adsorbed ions on the surface, as originally envisioned by Helmholtz [44]. The Stern layer is where electrochemical reactions, such as ion dissociation (setting the equilibrium charge on a dielectric) and/or redox couples (setting the Faradaic current at an electrode), are believed to occur, within a molecular distance of the solid surface [59, 60]. The Stern layer capacitance (see below) helps to relieve the overcharging of the diffuse layer in Gouy-Chapman theory, but, due to steric constraints, it too cannot conceivably withstand a voltage much larger than $\Psi_{c}$. Instead, at larger voltages, the region of ion accumulation must inevitably extend away from the surface into the solution, where ions undergo hindered transport in a concentrated solution without having specific interactions with the solid.

The most basic aspect of a concentrated solution is the finite molecular length scale, $a_{0}$. Over half a century ago, Wicke and Eigen [31, 32, 97] made perhaps the first attempts to extend dilute solution theory to account excluded volume effects in a simple statistical mechanical treatment. The theory was developed further in the past decade by Iglic and Kral-Iglic 14, 15, 48, 54] and Borukhov, Andelman, and Orland [17, 18, 19]. These authors proposed free energy functionals, based on continuum (mean-field) approximations of the entropy of equalsized ions and solvent molecules, which they minimized to derive modified Poisson-Boltzmann equations (MPB). The motivation for this work was mainly to address the effect of large ions, whose sizes may be comparable to the screening length, in an equilibrium diffuse layer with $\Psi_{D} \approx \psi_{T}$. Our main point here is that crowding effects can also be very important for small ions near a polarizable surface, when subjected to a "large" voltage the exceeding the threshold $\Psi_{c}$. No matter how dilute is the bulk solution, a sufficiently large electric field can always draw enough ions to the surface to create a concentrated solution in the diffuse layer.

There have also been attempts to go beyond the meanfield approximation of steric effects, by treating specific (and in some cases also Coulombic) ion-ion and ion-wall interactions. Simple MPB equations have been proposed, which modify the charge density to account for the spatial correlation function between an ion in solution and a flat wall (via an effective external potential) based on molecular dynamics simulations [33, [50, 76], but such models are not easily extended to any other geometry, such as a rough wall [53]. Corrections of this type can also be obtained from a general probabilistic model of interacting ions, whose dynamics are given by nonlinearly coupled Langevin equations. Using this approach, Schuss, Nadler and Eisenberg rigorously derived "condi- 
tional PNP" (and PB) equations where each ion concentration $c_{i}(\mathbf{r})$ in the mean-field Poisson's equation (2) is replaced by the conditional probability density of finding an ion at a certain position, given the positions of the other ions [67, 68, 86], although a simple closure of the model requires further assumptions about statistical correlations.

There are a variety of general statistical mechanical approaches from liquid state theory [45], which have been applied to electrolytes, taking into account not only steric effects, but also many-body electrostatic correlations (see below). Since the 1970s, the modest, but challenging, goal has been to accurately predict the equilibrium distribution of ions in Monte Carlo simulations of the "primitive model" of charged hard spheres in a homogeneous dielectric continuum bounded by a hard, charged wall. Typically, the model is studied in the limit of "small" surface potentials $\left(\Psi_{D} \approx k T / e\right)$ relevant for equilibrium surfaces. For example, a plethora of MPB equations (such as "MPB4", "MPB5",...) perturbing Gouy-Chapman theory have been derived by variations on the Mean Spherical Approximation [21, 55, 56, 73, 74, 75, 84]. More complicated, but often more accurate, theories have also been derived using statistical Density Functional Theory (DFT) [38, 39, 49]. By writing the ion flux as the gradient of an electrochemical potential obtained from the DFT free energy functional [37] (as we do in Part II, using the much simpler functional of Borukhov et al. [18]), it has been shown that the selectivity of ion channels can be understood to a large extent in terms of entropic effects of charged hard spheres [80].

In spite of many successes and the appealing firstprinciples nature of liquid-state theories, however, they are not ideally suited for our purpose here of capturing time-dependent, large-voltage effects in a simple model. Liquid-state theories are not analytically tractable and thus not easily coupled to macroscopic continuum models, although perhaps empirical fits could be developed for quantities of interest. For example, the DFT approach requires nontrivial numerical methods, just to evaluate the steady flux in a one-dimensional ion channel [37, 80]. Another concern is that liquid-state theories have been designed and tested only for "small" voltages $\left(\Psi_{D} \approx k T / e\right)$ at equilibrium surfaces, so it is not clear how well they would perform at large, time-dependent voltages, since there are no rigorous error bounds. For example, the double-layer differential capacitance in modified PB theories often increases with voltage [73], sometimes even faster than in PB theory [84], but we will argue below that it must decrease at large voltages, especially in concentrated solutions. In light of the scaling $e \Psi_{D} / k T$ from PB theory (3), a related problem is that, until recently [49, 55], most theories have been unable to predict the decay of capacitance at low temperature in concentrated solutions. Although liquid-state theories for large voltages should certainly be developed, we will focus on much simpler mean-field, continuum descriptions of steric effects, in the hope of at least capturing some qualitative features of nonlinear dynamics analytically, across a large range of applied voltages

Consistent with this approach, we will also work with the mean-field continuum description of electrostatic interactions (2), which neglects discrete many-body correlations. In passing, we point the reader to some of the extensive literature on corrections to $\mathrm{PB}$ electrostatics, for the simplest possible model of an equilibrium liquid of point-like charges in a uniform dielectric medium. In the absence of specific interactions (such as steric repulsion), the fundamental length scale for ion-ion correlations is the Bjerrum length $(\approx 7 \AA$ in water at room temperature),

$$
l_{B}=\frac{e^{2}}{4 \pi \varepsilon k T}
$$

at which the bare Coulomb energy balances the thermal energy. Interesting consequences of many-body electrostatics, not present in the mean-field PB description, include Oosawa-Manning condensation of counterions around charged rods in polyelectrolytes [64, 71, 72], renormalized charge of colloidal spheres [2, 8, 9], enhanced counterion localization around discrete surface charges [46], and counterion-mediated attraction of likecharged objects [42, 43, 65]. The latter phenomenon is believed to be responsible for the condensation of DNA in multivalent electrolytes [12], as well as the adhesion of cement plaste [42, 52]. A key part of the physics is the attraction between an ion and its "correlation hole" resulting from a fluctuation, which has recently been incorporated into a modified PB equation for a flat wall [85]. In all of these problems, however, the equilibrium surface charge is typically small (up to a monolayer of ions); it would be interesting to study electrostatic correlations at a much more highly charged surface, such as an electrode applying a large voltage, $V \gg \psi_{T}$ (our focus here).

Finally, we mention solvent effects, which are much less studied, and surely also very important at large voltages. Electrochemists widely believe that water dipoles in the Stern layer are so highly aligned by large electric fields that the effective permittivity drops by an order of magnitude (e.g. from $80 \varepsilon_{0}$ to $5 \varepsilon_{0}$ ) [13]. At large applied voltages, where typical fields are of order $\mathrm{V} / \mathrm{nm}$, it is reasonable to expect that the reduced permittivity would extend into the diffuse layer. This could have a major effect on ion-ion correlations, since the Bjerrum length $l_{b} \propto \varepsilon^{-1}$ could get as large as $10 \mathrm{~nm}$. Other aspects of water structure, such as hydrogen bonded networks, could also be altered by large electric fields and large ion concentrations. It would be interesting to perform $a b$ initio quantum-mechanical simulations of highly charged double layers to investigate such effects, beyond the primitive model. 


\section{Scope of the present work}

In spite of the considerable literature on MPB descriptions of electrolytes in equilibrium or in steady state conduction, we are not aware of any attempt to go beyond dilute solution theory (PNP equations) in analyzing the dynamics of electrolytes in response to time-dependent perturbations, such as AC voltages. Accordingly, here we develop only some very simple models with the goal of identifying generic new features of diffuse-charge dynamics in a concentrated solution. As such, it is preferrable to start with equations that capture the essential physics, while remaining analytically tractable, at least in some representative cases. For this reason, we focus on meanfield theories of steric effects and specifically build on the MPB equation of Borukhov et al., which can be integrated analytically in a few simple geometries 17, 18, 19. Such models also make reasonable predictions across the entire range of voltages.

The contribution is broken into two parts. Here, in Part I, we consider the canonical problem of charging a thin double layer, viewed as an effective circuit element [6]. We begin in section II by describing two simple models for steric effects in a quasi-equilibrium diffuse layer: (i) A "composite layer" model, consisting of a dilute $\mathrm{PB}$ outer region and, at high voltage, an inner "condensed layer" of ions packed at the steric limit, and (ii) the MPB model of Borukhov et al., which decribes the same transition in a continuous fashion. In section III, we then analyze the diffuse-layer capacitance and its role in equivalent circuit approximations. In section IV, we calculate steric effects on salt adsorption and tangential surface conduction by the diffuse layer, and discuss how these high-voltage effects affect and modify the applicability of circuit models. In section V] we also briefly discuss the effects of a compact dielectric layer (which could model a Stern layer of adsorbed ions, or, more accurately, a thin film coating the solid), before concluding in section VI.

In Part II, we consider explicitly time-dependent problems with a general formalism, not only applicable to thin double layers. We start with the free energy functional of Borukhov et al. and derive modified Poisson-NernstPlanck equations (mPNP), based on a simple generalization of chemical potential (1) for concentrated solutions. As an illustration, we then repeat the nonlinear asymptotic analysis of Ref. [6] for the response of a blocking electrochemical cell (no reactions at the electrodes) to a suddenly applied voltage, to expose some general consequences of steric effects at large voltage. We also clarify the range of validity of the thin-double-layer circuit approximations derived here in part $\mathrm{I}$.

\section{TWO MODELS OF STERIC EFFECTS IN A THIN DIFFUSE LAYER}

We focus on the response of a thin diffuse layer to an applied voltage, where it suffices to consider only quasiequilibrium properties [6], as we justify carefully in Part II. Following Gouy and Chapman, we consider the case of a symmetric $z: z$ electrolyte here, although our reasoning is readily extendible to the general case. We also assume that the permittivity $\varepsilon$ is constant in space, which is certainly not correct when dense layers of ions form close to the surface. However, this can be taken into account in a following step and does not change the qualitative picture emerging from the following analysis. We will return to this point below in section $\mathrm{V}$

There are at least three important lengths in our models. The first is the Debye length $\lambda_{D}$ given by (5), which sets the width of the diffuse layer at low voltage and low bulk concentration, $c_{0}$. The second is the mean spacing of ions in the bulk electroylte, $l_{0}=\left(2 c_{0}\right)^{-1 / 3}$, and the third is the mean spacing of ions (essentially of the same sign) at the maximum concentration, $a=c_{\max }^{-1 / 3}$. A fourth scale $L$ would characterize the geometry, as in Part II, but here we consider the regime of thin double layers, where $\lambda_{D} \ll L$. A fifth scale would be the Bjerrum length $l_{B}$, which we neglect by making the usual mean-field approximation.

From the first three lengths, there are two dimensionless groups. The first is $a / \lambda_{D}$, which we assume to be small for simple electrolytes in somewhat dilute solutions, so that steric effects are important only very close to the surface, at the inner portion of the diffuse layer (and even then, only at large voltages). The second dimensionless group can be written as the mean volume fraction of ions in the bulk,

$$
\nu=2 a^{3} c_{0}=\left(a / l_{0}\right)^{3}
$$

a natural measure of non-diluteness, which controls the importance of steric effects, along with the dimensionless voltage, $z e \Psi_{D} / k T$. In the figures below, we display results for $\nu=0.00005,0.005$, and 0.5 to span the range from dilute to highly concentrated solutions.

We stress that the phenomenological parameter $a$ is not necessarily the diameter of an ion, $a_{0} \approx 1 \AA$. We prefer to think of it as a cutoff for the unphysical divergences of PB theory, which we know must exist, and our goal is to understand its consequences. This cutoff length could include at least a solvation shell, e.g. $a \approx 3 \AA$, but ion-ion correlations could effectively increase it further, since the Bjerrum length is at least $7 \AA$. As noted above, taking into account the decrease of permittivity (by as much as a factor of ten) or other solvent effects at large fields could make $l_{b}$, and thus perhaps also $a$, as large as $10 \mathrm{~nm}$. As a guide to using our results, we refer to Fig. 1 for the value of the dimensionless parameter $\nu$ for different values of $a$ and $c_{0}$. 


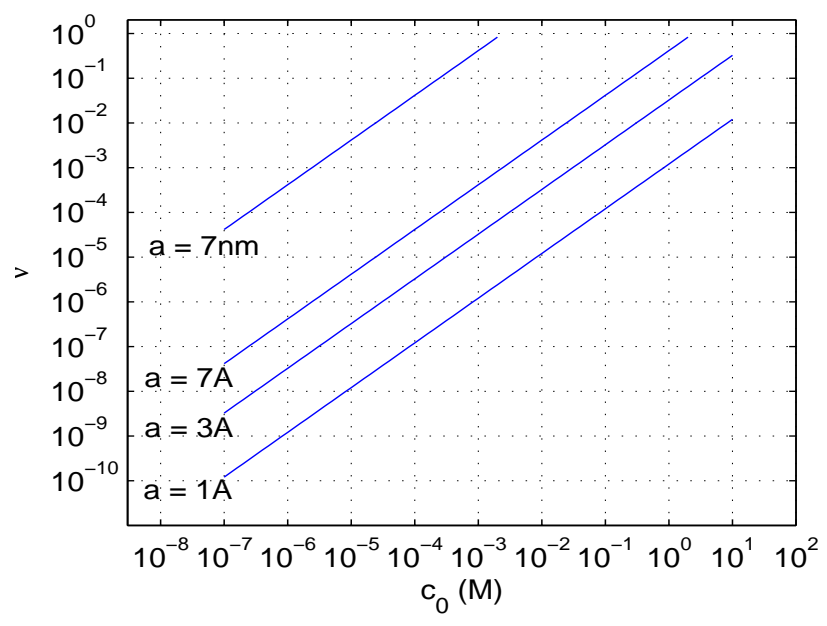

FIG. 1: The dependence of the dimensionless parameter $\nu$ as a function of the bulk concentration $c_{0}$ and effective ion size $a$.

\section{A. A composite diffuse-layer model}

In this model, we assume that the concentration fields are governed by Boltzmann distributions

$$
c_{ \pm}=c_{0} e^{\mp z e \psi / k T}
$$

wherever they are meaningful, that is, whenever these concentrations are weaker than a physical limit $1 / a^{3}$, which is set by the ion size. For both ion species, if the formula (12) yields a quantity bigger than $1 / a^{3}$, we set the concentration field of the counter-ion to be $1 / a^{3}$, and assume that the coions are excluded from the corresponding condensed layer. The basic physics is shown in Fig. 2]

For most geometries, such as rough surface, this model is ill-posed and would require additional constraints to determine the location the sharp free boundary separating the "dilute" Boltzmann region and the "condensed" region at the maximum density. For a flat, semi-infinite diffuse layer with a given total charge $q_{D}$ or total voltage $\Psi_{D}$, however, the transition occurs at a single well defined position, $x=l_{c}$, away from the solid surface (at $x=0)$. In that case, we separate the diffuse-layer into two parts if the potential is strong enough

$$
\begin{array}{ll}
c_{ \pm}=c_{0} e^{\mp z e \psi / k T} & \text { if } a^{3} c_{ \pm}<1, x>l_{c} \\
c_{ \pm}=a^{-3}, c_{\mp}=0 & \text { if } a^{3} c_{0} e^{\mp z e \psi / k T} \geq 1,0<x<l_{c}
\end{array}
$$

Note that in this simplistic dichotomy the concentration field for co-ions is slightly discontinuous at the critical potential mentioned earlier,

$$
\Psi_{c}=\frac{k T}{z e} \ln \frac{2}{\nu}
$$

where again $\nu=2 a^{3} c_{0}$ is volume fraction occupied by (all) ions at zero potential in the bulk. Although very

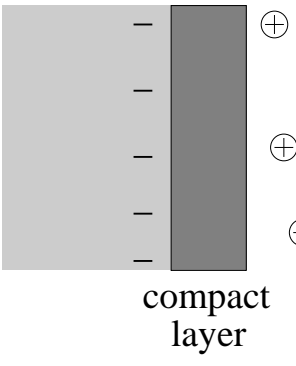

(a)
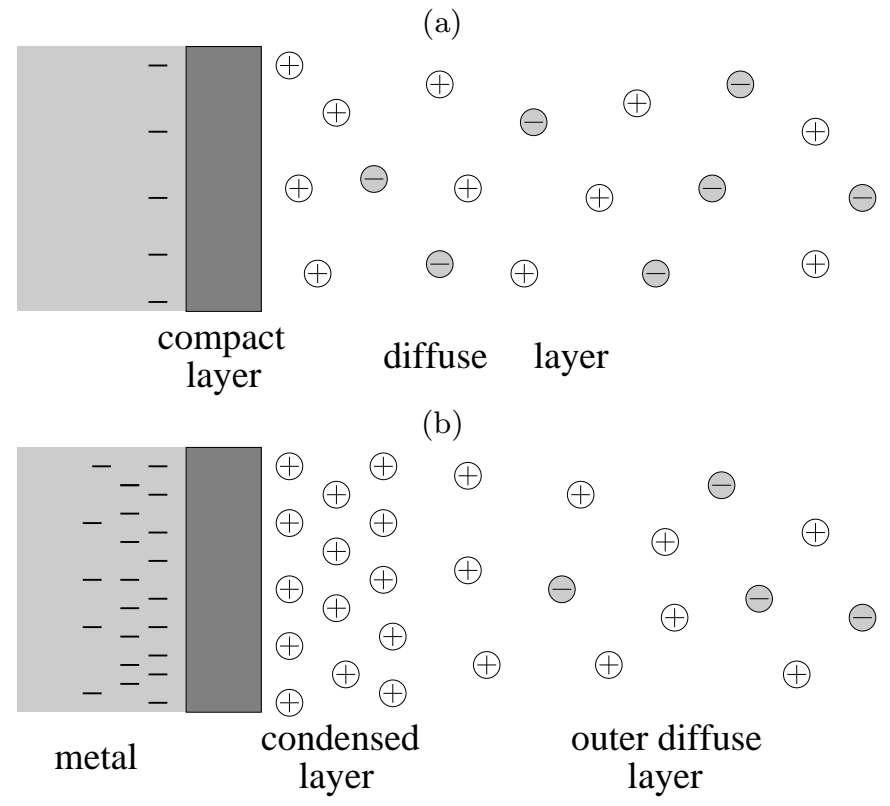

(b)

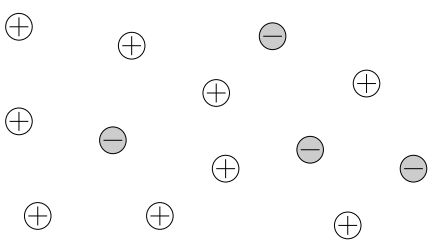

outer diffuse
layer

FIG. 2: (a) Most prior work in electrokinetics has dealt with surfaces of pre-existing equilibrium charge at the scale of one electron per surface atom or less. This charge can be screened by roughly a monolayer of ions (partly in the diffuse layer) which corresponds to a "small" double layer voltage, of order $\psi_{T}=k T / e$. (b) In contrast, nonlinear electrokinetics deals with polarizable (mainly metal) surfaces, where much higher surface charge densities can be produced by an applied voltage or electric field nearby, and thus the double layer can "overcharge" to the point where diluite-solution theory no longer applies. The existence of a minimum ion spacing implies the formation of a condensed layer of counterions near the surface.

simple, this model already captures steric effects at large voltages to a great extent.

Let us compute the thickness $l_{c}$ of the corresponding layer of "condensed" counter-ions. We consider an electrode to which a strong negative potential $\Psi_{D}$ is applied to the diffuse layer with respect to the bulk, such that $\left|\Psi_{D}\right|=-\Psi_{D}>\Psi_{c}$ which leads to a condensed layer of positive ions in its vicinity.

Poisson's equation for the thin diffuse layer reads

$$
\varepsilon \frac{d^{2} \psi}{d x^{2}}=-\rho
$$

since (for now) the permittivity $\varepsilon$ is assumed constant. Within the condensed layer, we have $\rho=z e c_{+}=z e / a^{3}$, so by integrating, we obtain

$$
\frac{d \psi}{d x}=-\frac{z e}{\varepsilon a^{3}} x+\frac{q_{c l}}{\varepsilon}, \psi=-\frac{1}{2} \frac{z e}{\varepsilon a^{3}} x^{2}+\frac{q_{c l}}{\varepsilon} x
$$

where $-q_{c l}$ is the surface charge density on the electrode, so that $q_{c l}$ is the total charge per unit area in the diffuse layer.

Within the outer diffuse layer, the standard PB equa- 


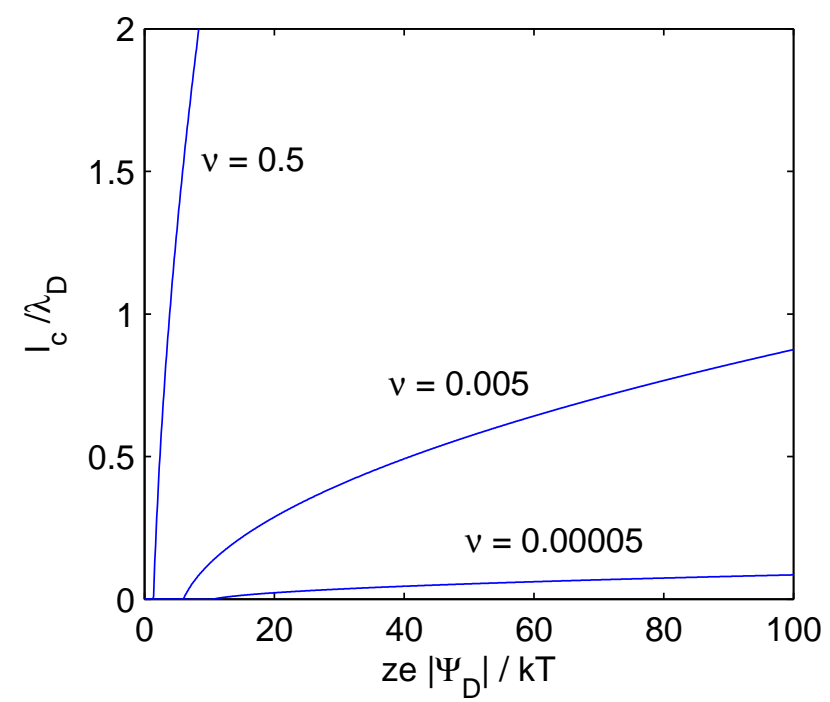

FIG. 3: The thickness of the condensed layer thickness $l_{c}$ as a function of the total voltage drop $\Psi_{D}$ across the diffuse and the condensed layers.

tion holds,

$$
\varepsilon \frac{d^{2} \psi}{d x^{2}}=2 z e c_{0} \sinh \left(\frac{z e \psi}{k T}\right)
$$

which gives

$$
\frac{d \psi}{d x}=-2 \sqrt{\frac{2 k T c_{0}}{\varepsilon}} \sinh \left(\frac{z e \psi}{2 k T}\right)
$$

At the interface between the condensed layer and the diffuse layer, we require the continuity of the electric field,

$$
q_{c l}=2 z e c_{0} \lambda_{D}\left(\sqrt{\frac{2}{\nu}}-\sqrt{\frac{\nu}{2}}\right)+\frac{z e}{a^{3}} l_{c}
$$

and of the electrostatic potential, so that

$$
-\Psi_{c}=-\frac{k T}{z e} \ln (2 / \nu)=-\left|\Psi_{D}\right|-\frac{1}{2} \frac{z e}{\varepsilon a^{3}} l_{c}^{2}+\frac{q_{c l}}{\varepsilon} l_{c}
$$

Combining these equations and solving for $l_{c}$ yields

$l_{c}=\lambda_{D} \sqrt{2 \nu}\left\{-1+\frac{\nu}{2}+\sqrt{\left(1-\frac{\nu}{2}\right)^{2}+\left[\frac{z e\left|\Psi_{D}\right|}{k T}-\ln (2 / \nu)\right]}\right\}$

which is plotted in Fig. 3 for several values of $\nu$. Generally, the condensed layer forms when the diffuse layer voltage $\Psi_{D}$ becomes only a few times the thermal voltage $k T / z e$, and then it grows sublinearly, proportionally to the square root of the potential drop as anticipated from Poisson's equation with a constant charge density.
From this equation and (19), we finally obtain the charge/potential relation for the composite diffuse layer:

$$
q_{c l}=2 z e c_{0} \lambda_{D} \sqrt{\frac{2}{\nu}} \sqrt{\left(1-\frac{\nu}{2}\right)^{2}+\left[\frac{z e\left|\Psi_{D}\right|}{k T}-\ln (2 / \nu)\right]}
$$

which holds for $\left|\Psi_{D}\right|>\Psi_{c}$, as assumed here. For weaker potentials, there is no condensed layer and the standard PB model holds:

$$
q_{p b}=-4 z e c_{0} \lambda_{D} \sinh \left(\frac{z e \Psi_{D}}{2 k T}\right)
$$

We note that, when compared to the PB charge density $q_{p b}$, the composite-layer charge density $q_{c l}$ is significantly reduced by the steric effects at higher $\Psi_{D}$. In particular, it increases only sublinearly, as opposed to exponentially in the PB model.

\section{B. The modified PB model}

The second model we consider is the classical meanfield description of steric effects in equilibrium mentioned in the introduction; we refer the reader to the literature for its derivation and statistical mechanical assumptions [14, 15, 17, 18, 18, 19, 31, 32, 48, 54, 97]. For a symmetric $z: z$ electrolyte, the concentrations in the diffuse layer as a function of the electrostatic potential with respect to the bulk $\psi$ are given by the modified Boltzmann distribution

$$
c_{ \pm}=\frac{c_{0} e^{\mp z e \psi / k T}}{1+2 \nu \sinh ^{2}\left(\frac{z e \psi}{2 k T}\right)}
$$

where the packing parameter $\nu=2 a^{3} c_{0}$ is again the bulk ion density scaled to its maximum value and $a$ is the effective size of the ions and the solvent molecules. Note that the concentration of each ion saturates and cannot exceed the steric limit.

In a mean-field approximation with these ion concentrations, the potential satisfies the modified PoissonBoltzmann (MPB) equation,

$$
\nabla^{2} \psi=\frac{z e c_{0}}{\varepsilon} \frac{2 \sinh \left(\frac{z e \psi}{k T}\right)}{1+2 \nu \sinh ^{2}\left(\frac{z e \psi}{2 k T}\right)} .
$$

Unlike the composite layer model, the MPB model can be applied to any geometry (just like the PB model). In the case of a flat diffuse layer, it gives a similar description, except that steric effects enter smoothly with increasing voltage, and there is no sharply defined condensed layer.

As for the first model, we can integrate the MPB equation across a thin double layer to obtain the normal electric field at the inner part of the diffuse layer

$$
\frac{d \psi}{d x}=-\operatorname{sgn}(\psi) \frac{2 z e c_{0} \lambda_{D}}{\varepsilon} \sqrt{\frac{2}{\nu} \ln \left[1+2 \nu \sinh ^{2}\left(\frac{z e \psi}{2 k T}\right)\right]}
$$




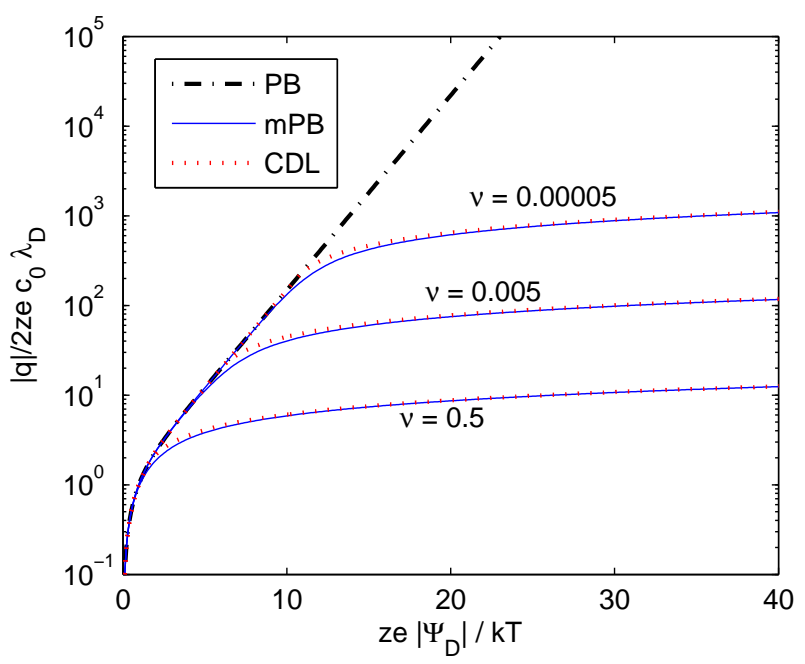

FIG. 4: The diffuse layer charge given by $\mathrm{PB}, \mathrm{MPB}$ and $\mathrm{CDL}$ models as a function of the across potential drop.

Integrating (15) using (26), we obtain for this model the relation between the charge per unit area in the diffuse layer $q_{m p b}$ and the potential drop across it, $\Psi_{D}$ :

$q_{m p b}=-\operatorname{sgn}\left(\Psi_{D}\right) 2 z e c_{0} \lambda_{D} \sqrt{\frac{2}{\nu} \ln \left[1+2 \nu \sinh ^{2}\left(\frac{z e \Psi_{D}}{2 k T}\right)\right]}$

This formula is illustrated in Fig. 4, and compared to the analogous formula for the composite diffuselayer model (22). Although the MPB concentrations (13) have been analyzed carefully by previous authors 14, 15, 17, 18, 18, 19, 48, 54], it seems the charge-voltage relation (27) has been overlooked, or at least is not stated explicitly in these papers. (Similarly, Chapman was the first to explicitly write down the formula (23) for the total charge [22], even though Gouy had thoroughly analyzed the concentration and potential profiles in the "Gouy-Chapman model" a few years earlier [41].)

We note in passing that the asymptotic behaviour at large voltages is similar for the two models presented, and corresponds to a layer of essentially constant charge density, which results using Poisson's equation in a total charge proportional to the square root of the potential drop.

\section{EQUIVALENT CIRCUIT ELEMENTS}

\section{A. A word on circuit models}

As widely recognized in electrochemistry [36, 63, 90], it is often appropriate to describe the effect of the dou- ble layers on the dynamic properties of a system through effective resistances and capacitances. The underlying assumption, is that the equilibration time of the double layer is fast compared to the dynamics of the global "circuit" considered, essentially because it is so thin so that transport processes within the double layer are rapid. A mathematical justification, starting from the simple PNP equations, can be given in terms of an asymptotic analysis of the thin double layer limit [ 6$]$.

Therefore, we anticipate that for the many situations where the double layer is thin compared to the system size, the dynamics can be understood to a significant extent using the equilibrium characteristics of the double layer. These then provide appropriate boundary conditions for the dynamic transport processes in the bulk.

In particular, the analysis presented in Ref. [6], shows that the capacitance, or more precisely the differential capacitance " $C$ ", of the double layer is a central quantity allowing the modeling of the system in terms of an "RC" circuit. The second quantity of relevance, as it characterizes the entrance into the strongly non-linear regime, is the neutral salt uptake by the double layer which can result in an appreciable depletion of the bulk, leading to modifications of its conductivity (affecting the resistance " $R$ " in the circuit) and thus of the dynamics. This paper and a subsequent one [24] also pointed out (in the context of dilute solution theory) that the tangential conduction through the diffuse layer is intimately tied to neutral salt adsorption, and indeed is governed by the same dimensionless "Dukhin number".

Therefore, we will proceed to compute all of these dynamical quantities for a thin quasi-equilibrium double layer, focusing on the general consequences of steric effects, which are common to the two models. After that, we will return to the question of surface capacitance and consider the effect of a thin dielectric layer (such as an oxide coating on a metal electrode, or perhaps a frozen Stern layer of adsorbed ions) on the overall dynamical response of the double layer.

\section{B. Total and differential capacitances}

The total capacitance of the diffuse layer can be obtained directly from the previous equations relating $q$ (the charge per unit area in the diffuse layer) to $\Psi_{D}$ (the voltage drop across the double layer), as simply $-q\left(\Psi_{D}\right) / \Psi_{D}$. We have already computed these quantities above, and they are compared to the $\mathrm{PB}$ result $(\nu=0)$ in Fig. 5. It is immediately obvious that the capacitance is greatly reduced at high voltage, compared to the predictions of dilute solution theory. The effect is so dramatic that the capacitance in both models reaches a maximum not much larger than the zero-voltage value, and even decreases for all values of voltage at sufficiently high concentration - the opposite trend from PB theory.

As noted above, the differential capacitance, defined 


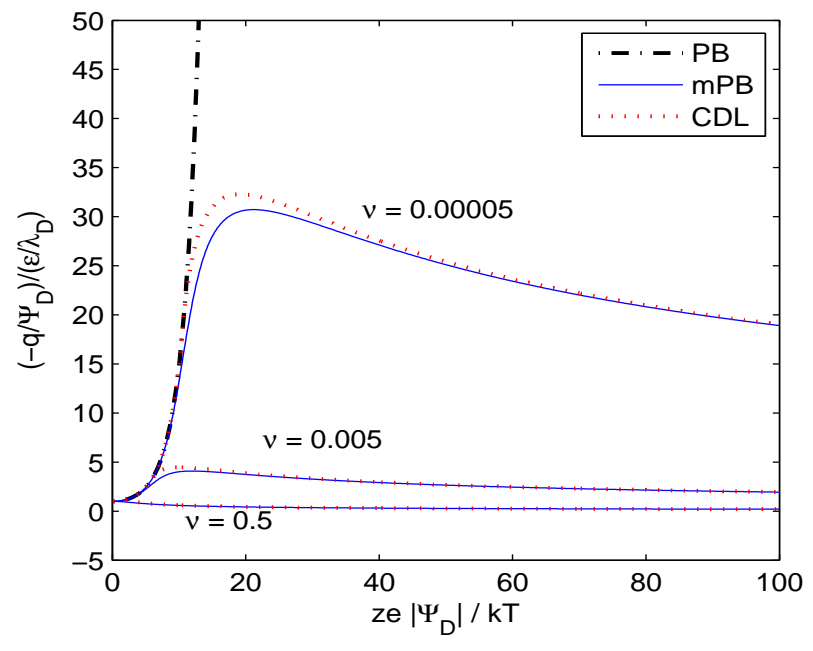

FIG. 5: The total capacitance $-q\left(\Psi_{D}\right) / \Psi_{D}$ of the diffuse layer as a function of $\Psi_{D}$.

for the diffuse layer as

$$
C_{D}\left(\Psi_{D}\right)=-\frac{d q}{d \Psi_{D}}
$$

is the relevant quantity for the dynamical response to an applied voltage. Throughout this paper, to be clear, we will use the notation $C$ only for the differential capacitance. In the PB model $(\nu=0)$, the differential capacitance from Eq. (23) has already been noted above:

$$
C_{D}^{\nu}=\frac{\varepsilon}{\lambda_{D}} \cosh \left(\frac{z e \Psi_{D}}{2 k T}\right) .
$$

For the composite diffuse layer (CDL) model introduced above, (19) yields

$$
C_{D}^{\nu}=\frac{\varepsilon}{\lambda_{D}} \frac{1}{\sqrt{2 \nu} \sqrt{\left(1-\frac{\nu}{2}\right)^{2}+\left[\frac{z e\left|\Psi_{D}\right|}{k T}-\ln (2 / \nu)\right]}}
$$

when $\Psi_{D}>\Psi_{c}$. Otherwise, the PB formula (29) still holds. For the MPB diffuse layer model, using (27), we find

$$
C_{D}^{\nu}=\frac{\frac{\varepsilon}{\lambda_{D}}\left|\sinh \left(\frac{z e \Psi_{D}}{k T}\right)\right|}{\left[1+2 \nu \sinh ^{2}\left(\frac{z e \Psi_{D}}{2 k T}\right)\right] \sqrt{\frac{2}{\nu} \ln \left[1+2 \nu \sinh ^{2}\left(\frac{z e \Psi_{D}}{2 k T}\right)\right]}}
$$

The different models are compared in Fig. 6 and Fig[7.

The qualitative trends in $C$ are again similar in both models of steric effects and dramatically different from PB theory. For both models, the differential capacitance increases at very low potentials (comparable to the thermal voltage, $k T / z e$ ) as it does in the PB model because steric effects are still negligible. These enter the picture at larger potentials and limit the storing capacity of the

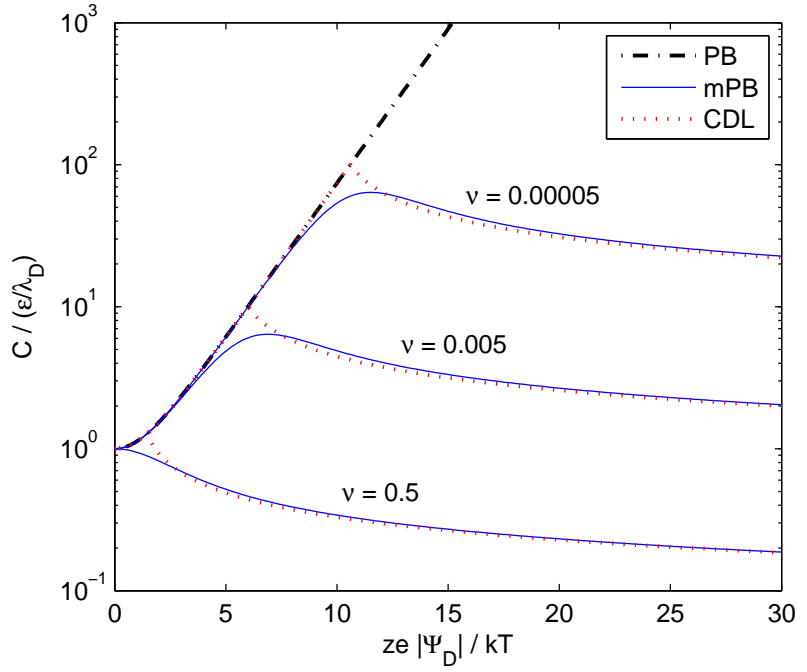

FIG. 6: The diffuse layer differential capacitance as a function of the potential drop across itself.

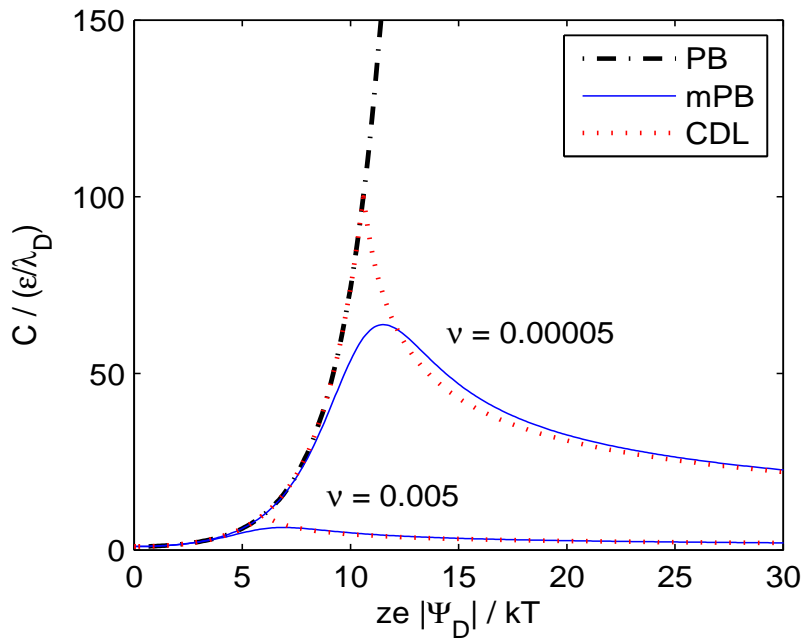

FIG. 7: Same as fig 6] except with a linear scale on the y-axis. The case $\nu=0.5$ is not shown.

layer, with a differential capacitance that actually decreases to zero at large potentials. As a consequence both models predict a non-monotonic differential capacitance, and show a maximum at intermediate values.

Of course, there are some clear differences in the detailed predictions of the two models, shown in Figs 6 and 7. Although the limiting behaviors at large and small voltage are similar, the transition is unphysically sudden in the CDL model, compared to the more reasonable, smooth transition in the MPB model. This is especially true at low concentrations, where the sudden, delayed appearance of steric effects in the CDL model gives rise to a sharp cusp in the differential capacitance versus voltage. 
At high concentrations, where the "low voltage" regime of dilute solution theory effectively vanishes, the CDL model also fails to predict the immediate onset of steric effects, even at small voltages, which causes a monotonic decrease in differential capacitance - the opposite trend of the PB model. Nevertheless, the CDL model manages to approximate the trends of the MPB model well, with an appealingly simple physical picture.

In summary, three basic features show up in both models, which we take as an indication that they qualitatively hold irrespective of the specific approximations embedded in each model: (i) the differential capacitance $C_{D}\left(\Psi_{D}\right)$ is weaker at moderate and high potentials than if steric effects are neglected (as in the PB scheme); (ii) at moderate concentrations, the differential capacitance varies non-monotonously with a peak at intermediate voltages and a slow decrease towards zero at higher voltages, (iii) at the steric limit $(\nu=1)$, the differential capacitance is a strictly decreasing function of voltage (in the MPB model). These effects are all explained by the strong tendency of ions to form a condensed inner layer at high voltage and/or high concentration, when steric effects are taken into account. This greatly reduces the differential (and total) capacitance compared to classical PB theory, which neglects the finite size of ions, and thus predicts an absurd exponential pile-up of ions extremely close to the surface (less than one molecular radius) in the nonlinear regime.

\section{Diffuse-layer charging dynamics}

These revelations have important consequences for our understanding of double-layer charging in many situations. In the simplest picture of an equivalent $\mathrm{RC}$ circuit, with $R$ the resistance of the bulk, these statements relate to the response time of the system to a step or an AC applied voltage. The typical response time for a driving of amplitude $V$ is $\tau_{c}(V)=R C_{D}(V)$, so the classical picture from PB theory (29) has been that nonlinearity greatly slows down the charging dynamics [6, 24, 58, 62, 88, 92$]$. Although this may occur in a dilute solution for a relatively small range of voltages (typically only several times the thermal voltage), steric effects in a concentrated solution bound the relaxation time at a value much less than expected from the PB model, in both the CDL and MPB models. This is clear in Fig. 8, where we solve the $\mathrm{RC}$ circuit dynamics

$$
C_{D}\left(\Psi_{D}\right) \frac{d \Psi_{D}}{d t}=\frac{V-\Psi_{D}}{R}
$$

for the three models numerically, to obtain the diffuselayer voltage $\Psi_{D}(t)$ in response to a suddenly applied voltage $V$ across the layer in series with a bulk resistance $R$. In addition, importantly, the response time of an electrolytic cell is found to decrease with the amplitude of the applied voltage above the threshold for strong steric effects, $V>\Psi_{c}$. As shown in Fig $8(\mathrm{~b})$, the relaxation is

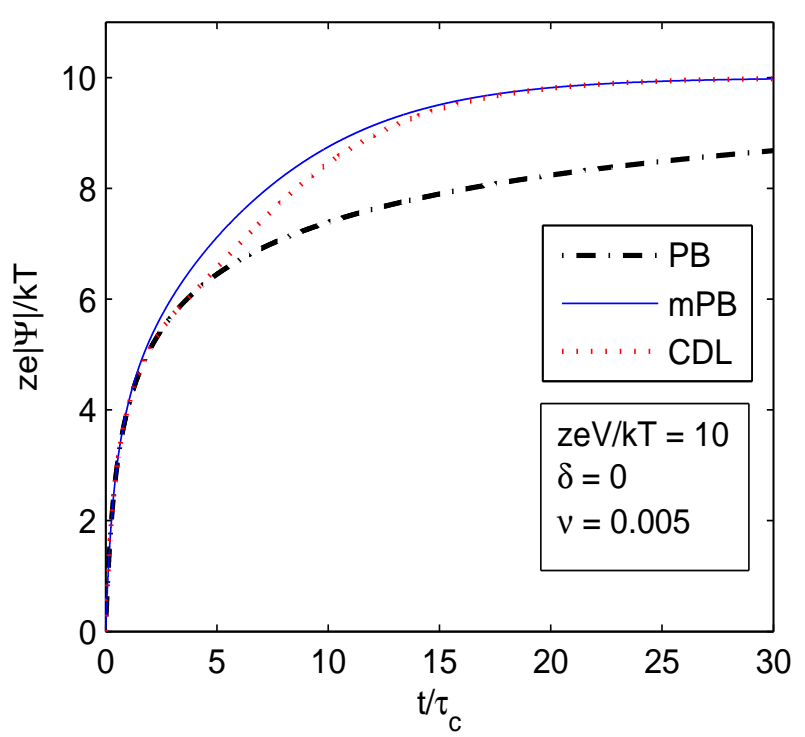

(a)

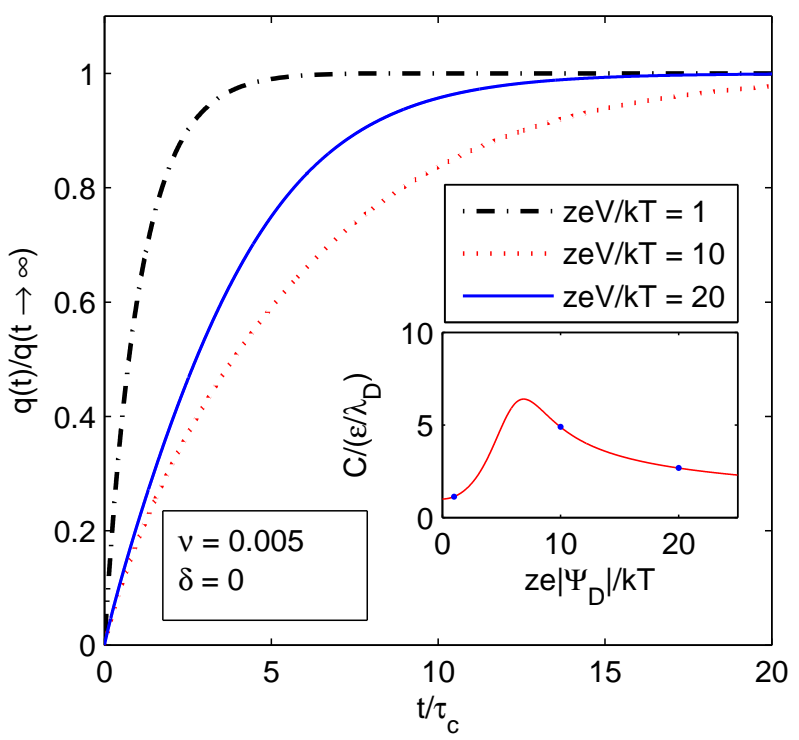

(b)

FIG. 8: Diffuse-layer relaxation in response to a sudden voltage step across a blocking cell of width $2 L$ and constant resistance $R$. Time is scaled with the charging time $\tau_{c}=\lambda_{D} L / D=R C\left(\Psi_{D}=0\right)(\delta=0)$. (a) Relaxation of the diffuse-layer voltage $\Psi_{D}$ in the PB, MPB, and CDL models for the same applied voltage, $V=10 k T / z e$. (b) Relaxation of the total diffuse-layer charge for different voltages, $z e V / k T=1,10,20$, in the MPB model with $\nu=0.005$, showing varying response times due to the non-monotonic voltage dependence of the differential capacitance (inset).

faster for $V=20 \mathrm{kT} / z e$ than for $10 \mathrm{kT} / z e$, since $\Psi_{c}=$ $8.3 k T / z e$ for $\nu=0.005$. 


\section{BEYOND CIRCUIT MODELS}

\section{A. Diffuse layer salt adsorption}

As recently pointed out in Ref. [6], circuit models can break down at large voltages if a large number of ions (mostly counter-ions) are engulfed by the diffuse layers with a resulting depletion of ions in the bulk. The total salt concentration in the diffuse layer (counter-ions plus co-ions) increases with voltage, regardless of the sign of the charge. Therefore, a diffusion layer forms and relaxes into the neutral bulk whenever a voltage is applied across a double layer at a blocking surface (although reactions and/or rejection of adsorbed ions from the Stern layer could lead to negative adsorption or salt expulsion in other situations [61]). In the absence of salt injection by the surface, the positive adsorption of neutral salt by the diffuse layer is present in the PB description, where the counter-ion concentrations increase exponentially with voltage [6]. It is still present but obviously weaker in models accounting for steric effects, which severely limit the capaticity of the diffuse layer to store additional ions at high voltage. We now quantify this statement using the two simple models introduced above.

Following notations introduced in Ref. [6], we define the excess neutral salt in the double layer as

$$
w=w^{\nu}=\int_{\text {surface }}^{\text {bulk }}\left(c_{+}+c_{-}-2 c_{0}\right) d x .
$$

For the PB model one finds [6],

$$
w^{\nu=0}=8 c_{0} \lambda_{D} \sinh ^{2}\left(\frac{z e \Psi_{D}}{4 k T}\right) .
$$

For the CDL model, the same equation holds for $\Psi_{D}<$ $\Psi_{c}$, while above this value $\Psi_{D}>\Psi_{c}$ we obtain

$$
w^{\nu}=\left(\frac{2}{\nu}-2\right) c_{0} l_{c}+2 c_{0} \lambda_{D}\left(\sqrt{\frac{2}{\nu}}+\sqrt{\frac{\nu}{2}}-2\right)
$$

with $l_{c}\left(\Psi_{D}\right)$ to be extracted from (21). For the MPB model

$$
w^{\nu}=\int_{0}^{\frac{z e \Psi_{D}}{k T}} \frac{(\cosh u-1)}{1+2 \nu \sinh ^{2} u} \frac{2 c_{0} \lambda_{D}(1-\nu) d u}{\sqrt{\frac{2}{\nu} \ln \left(1+2 \nu \sinh ^{2} u\right)}}
$$

which we can compute numerically.

Unlike the PB formula (33), which predicts exponentially diverging salt uptake for increasing $\Psi_{D}$, the steric modified formulae (34) and (35) predict sublinear (square-root like) dependence on $\Psi_{D}$, at large voltages, as can be seen by inspection of (33)- 35 or from the plots in Fig. 9. This can be understood qualitatively as a consequence of the roughly square-root voltage dependence of the condensed layer width (due to its constant charge density), since most of the adsorbed ions are condensed counterions at large voltages. (See Fig. 3 and Eq. (21)

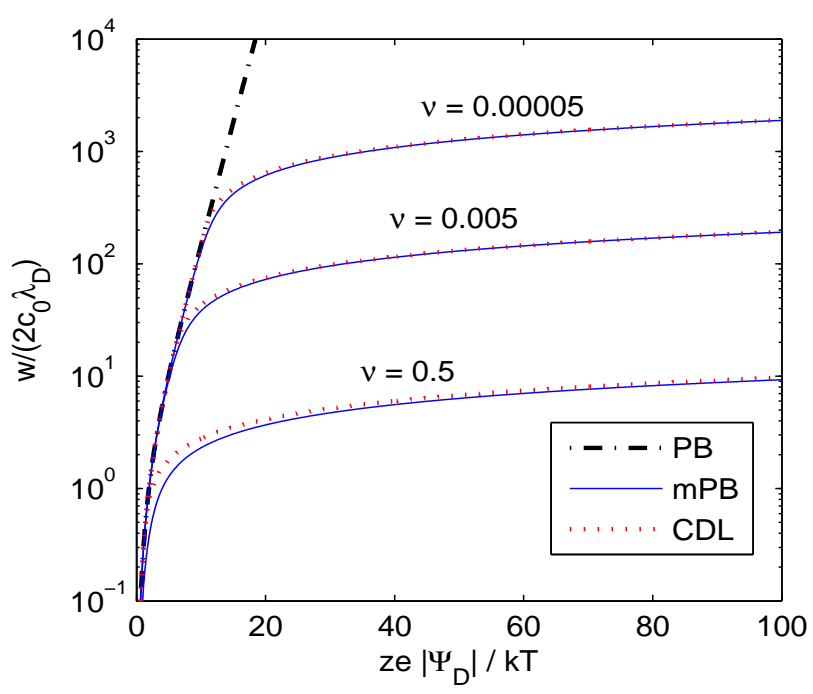

FIG. 9: The diffuse layer neutral salt uptake $w$ as a function of the potential difference $\Psi_{D}$.

for the CDL model.) Another way to see it in both models is that the total salt adsorption is asymptotic to the total charge, $w \sim q / z e$, which is true for any nonlinear double layer model (PB, MPB, CDL,...) since the coion density goes to zero across most of the diffuse layer at large voltages. As explained in Ref. [6], in response to an applied voltage the neutral bulk electrolyte becomes depleted, as the electric field draws counterions into the diffuse layer and conducts coions away through the bulk, resulting in a slowly expanding diffusion layer, reducing the bulk concentration accordingly.

The main feature, again common to both models, is that the salt uptake at large voltage is greatly reduced in comparison to the exponential growth predicted by the PB picture. This is important for the dynamics as this quantity sets the limit of applicability of the widely used RC circuit model [6]. This equivalent circuit approximation should thus hold up to much larger values of potential when steric effects are included.

\section{B. Breakdown of circuit models}

With analytical expressions for total salt adsorption by the diffuse layer, we can estimate the upper limits on the applied voltage where the circuit approximation breaks down in the various models. For an electrolyte cell of thickness $2 L$, the salt uptake by the diffuse layer corresponds to a removal of $2 w$ charge carriers (ions) per unit surface, from a bulk that contained $2 c_{0} L$ such carriers initially. As long as the ratio of these two quantities $w /\left(c_{0} L\right)$ is small, the total resistance of the $\mathrm{RC}$ circuit is roughly unaffected by the salt adsorption. So, an esti- 
mate for the limit is [6]

$$
\alpha_{s}=\frac{w}{c_{0} L} \ll 1,
$$

which translates an upper bound on the applied voltage, $|V|<V_{\text {threshold }}$ for the $\mathrm{RC}$ description to remain valid (in the thin double layer limit $\lambda_{D} / L \ll 1$ ). For the $\mathrm{PB}$ model, the upper bound,

$$
V_{\text {threshold }} \approx \frac{2 k_{B} T}{z e} \ln \left(\frac{L}{4 \lambda_{D}}\right), \text { (dilute) }
$$

is not much larger than the thermal voltage, due to the weak logarithmic dependence on $L / \lambda_{D}$.

For the models accounting for steric effects, however, the upper bound is greatly increased in concentrated solutions,

$$
V_{\text {threshold }} \approx \frac{k_{B} T}{z e}\left(\frac{L}{\lambda_{D}}\right)^{2} a^{3} c_{0} \text { (non-dilute) }
$$

This shows that the widely used circuit approximation for a thin double layer $\lambda_{D} \ll L$ does not break down until enormous voltages, $V \propto\left(L / \lambda_{D}\right)^{2}$, in a concentrated solution. In a very dilute solution, where $\nu=2 a^{3} c_{0} \ll 1$, the circuit approximation may break down at moderate voltages, but only in a microsystem, where the double layers are not so thin. For example, even in an (aqueous) electrolyte with $\lambda_{D}=10 \mathrm{~nm}, c_{0}=10^{-3} \mathrm{M}, a=5 \AA$ and in a microdevice with $L=100 \mu \mathrm{m}$ features, the threshold voltage (with steric effects) is roughly 0.2 Volts.

This estimate, however, neglects the possibility of a transient breakdown of the RC circuit approximation, prior to diffusive relaxation across the entire cell. In the case of response to a suddenly applied DC voltage, the salt adsorption by each the diffuse layer occurs over a time scale, $\tau_{c}=\lambda_{D} L / D$, during which diffusion spreads the nearby region of depleted neutral bulk solution over a distance $\sqrt{D \tau_{c}}=\sqrt{\lambda_{D} L}$. Therefore, the requirement that the local bulk conductivity does not change significantly during charging dynamics yields the refined estimate [6],

$$
\alpha_{d}=\frac{w}{c_{0} \sqrt{\lambda_{D} L}} \ll 1
$$

which replaces $L / \lambda_{D}$ by $\sqrt{L / \lambda}$ in the estimates above. This sets a lower bound for the limiting voltage for the validity of circuit models.

In the case of $\mathrm{AC}$ forcing at frequency $\omega$, the bound $\alpha_{d} \ll 1$ is appropriate for low frequencies, $2 \pi \omega \tau_{c} \ll 1$, but circuit models remain valid up to higher voltages at higher frequencies. At moderate frequencies, $2 \pi \omega \tau_{c}>1$, the double layer does not have enough time for complete charging, and the Warburg-like diffusion layer due to salt adsorption (which oscillates at twice the frequency) only propagates to a distance, $\sqrt{D / 4 \pi \omega}$. Therefore, we may crudely estimate $\left(w / c_{0}\right) \sqrt{4 \pi \omega / D} \ll 1$ to avoid significant changes in bulk conductivity in the diffusion layer.
(A more careful estimate would take into account that only partial charging and salt adsorption occur with increasing frequency.) At higher frequencies, $\omega \lambda_{D}^{2} / D \approx 1$, the diffuse layer does not have enough time to equilibrate, and little charging occurs.

These arguments can be made more precise using matched asymptotic expansions to describe the thin double layer limit, starting from an explicitly time-dependent model. For the case of one-dimensional response to a suddenly applied voltage, this was done in Ref. [6] starting from the Poisson-Nernst-Planck equations of (timedependent) dilute solution theory. In part II, we will derive modified PNP equations for dynamics with steric effects and repeat the same kind of asymptotic analysis to reach a similar conclusion our simple arguments here: Steric effects greatly extend the range of applicability of $\mathrm{RC}$ circuit models, compared to what would be expected on the basis of dilute solution theory.

\section{Diffuse-layer surface conduction}

Another feature not present in circuit models is the possibility of current passed along the surface through the diffuse layer, demonstrated by Bikerman [10] and considered extensively in theories of electrokinetics by Dukhin and collaborators 30]. As first shown in Ref. [6] and elaborated in Ref. [24] in the setting of dilute solution theory, the relative strengths of tangential "surface fluxes" through the diffuse layer, compared to bulk fluxes, are controlled by the same dimensionless groups that govern ion adsorption (discussed above). This is actually quite a general result, as we now briefly explain. We will thus conclude that steric effects also greatly reduce the importance of surface conduction in the diffuse layer compared to the classical predictions of dilute solution theory.

Assuming small local perturbations from thermal equilibrium, the flux density (number/area-time) of ionic species $i$ is given by

$$
F_{i}=-b_{i} c_{i} \nabla \mu_{i}
$$

where the chemical potential $\mu_{i}$ generally has a different form than (11) in a concentrated solution (e.g. see Part II). Consider a thin diffuse layer near a charged surface, where the ion concentration $c_{i}$ departs from its nearby neutral bulk value $c_{i}^{b}$. Due to fast relaxation at the small scale of the screening length, the diffuse-layer concentration remains in quasi-equilibrium at nearly constant chemical potential in the normal direction, $\mu_{i} \sim \mu_{i}^{b}$, in the thin double layer limit. There can, however, be small tangential gradients, $\nabla_{\|} \mu_{i} \neq 0$ at the macroscropic length scale leading to an excess diffuse-layer "surface" 
flux density (number/length·time):

$$
\begin{aligned}
F_{i}^{s} & =\int_{\text {surface }}^{\text {bulk }}\left(-b_{i} c_{i} \nabla_{\|} \mu_{i}+b_{i} c_{i}^{b} \nabla_{\|} \mu_{i}^{b}\right) d x \\
& \sim-\nabla_{\|} \mu_{i}^{b} \int_{\text {surface }}^{b u l k} b_{i}\left(c_{i}-c_{i}^{b}\right) d x
\end{aligned}
$$

For a constant mobility $b_{i}$, this takes the same form as the bulk flux density (37),

$$
F_{i}^{s}=-b_{i} \Gamma_{i} \nabla_{\|} \mu_{i}
$$

where the bulk concentration (number/volume) has been replaced by the diffuse-layer "surface concentration" (number/area),

$$
\Gamma_{i}=\int_{\text {surface }}^{b u l k}\left(c_{i}-c_{i}^{b}\right) d x
$$

In a concentrated solution, we generally expect that the mobility $b_{i}$ might decrease in the difuse layer, due to steric effects and large normal electric fields, so this formula may overestimate the surface flux density.

Following Bikerman 10, 11, 24], we may estimate the relative importance of surface to bulk flux densities at a length scale $L$ by the dimensionless group

$$
\frac{F_{i}^{s}}{F_{i}^{b} L}=\frac{\Gamma_{i}}{c_{i}^{b} L}
$$

which we see also measures the relative importance of "surface adsorption" of ion $i$ in the diffuse layer relative to the bulk concentration. For a highly charged diffuse layer $\left|\Psi_{D}\right| \gg \Psi_{c}$, the ions are mostly of one type (counterions), so $\Gamma_{i} \sim q \sim w$. The surface current is also carried mostly by those ions, $J^{s} \sim z e F_{i}^{s}$, while the bulk current is carried by both ions, $J^{b} \approx 2(z e) F_{i}^{b}$ (neglecting diffusion compared to electromigration). Therefore, we see that the "Dukhin number" comparing surface conduction at a highly charged diffuse layer to bulk conduction at nearly uniform concentration,

$$
\mathrm{Du}=\frac{J^{s}}{J_{b} L} \sim \frac{w}{2 c_{0} L}=\frac{\alpha_{s}}{2}
$$

is roughly half of the dimensionless group $\alpha_{s}$ governing salt adsorption by the diffuse layer.

We have seen that steric effects greatly reduce $w$ compared to the predictions of dilute solution theory, so that $\alpha_{s}=O\left(\lambda_{D} / L\right)$ remains small up to rather high voltages. Since the calculation above over-estimates the importance of surface conduction, it is clear that steric effects also greatly reduce the Dukhin number compared to the predictions of dilute solution theory. We conclude that surface conduction in a thin diffuse layer does not become important until voltages large enough to violate the equivalent circuit approximation are applied across it. Compact layer surface conduction may still be important in some cases, but it too is limited by the same steric effects.

\section{COMPACT LAYER EFFECTS}

We now check the robustness of these conclusions to the additional presence of an insulating surface layer between the metal electrode and the electrolyte where the EDL models are applied. We suppose that this layer is not involved in the dynamics so that its properties do not change, and assume for the sake of simplicity that these properties are linear so that it can be described as a fixed surface capacitance $C_{s}$.

Such models have been used in many circumstances, sometimes to describe the Stern layer corresponding to condensed ions. Our approach here is different in the sense that a layer of condensed ions would be involved in the charging/discharging process. What we have in mind is closer to a thin film, e.g. of oxide on the metal, of thickness $h_{s}$ and dielectric constant $\epsilon_{s}$ so that $C_{s}=\epsilon_{s} / h_{s}$. This form has been proposed to model coating layers on electrodes in the context of AC electrokinetics [1].

The overall differential capacitance of the interface is now

$$
\frac{1}{C}=\frac{1}{C_{D}}+\frac{1}{C_{S}}
$$

with $C_{S}=d q / d \Psi_{S}$, where $q$ is the total charge per area in the double layer, and $\Psi_{S}$ is the voltage drop across the aforementioned compact layer. The total voltage drop across the interface is $\Psi=\Psi_{S}+\Psi_{D}$, and $q / \Psi$ is the total capacitance of the interface.

A useful dimensionless parameter to quantify the effect of this surface layer is $\delta=\varepsilon /\left(\lambda_{D} C_{S}\right)$, which has been employed recently in general studies of diffusecharge dynamics [6, 16], as well as in theoretical models [1, 5, 40, 58, 78, 92] and in the fitting of experimental data 57, 77] for induced-charge (AC or DC) electrokinetics. No surface layer corresponds to $\delta=0$. In the above mentioned picture of the layer of oxide, $\delta=\left(\varepsilon / \epsilon_{s}\right)\left(h_{s} / \lambda_{D}\right)$ so that even very thin oxide layers can yield not too small values of $\delta$ if $\varepsilon / \epsilon_{s}$ is large.

Let us now compare the differential capacitance $C$ (with surface layer) to the bare double layer differential capacitance previously plotted in Fig 7 for the PB equation and the two models with steric effects. The corresponding plots are provided on Fig 10 for $\delta=0.25$.

Many qualitative points are obvious. First, the PB differential capacitance does not blow up exponentially anymore, as the surface layer takes over when the double layer voltage gets large, so that $C$ converges to the finite value $C_{s}$ within the $\mathrm{PB}$ model. Most of the potential drop is then across the surface layer. Second, the two main consequences of steric effects pointed above remain valid: $C$ is weaker when steric effects are taken into account, and $C(\Psi)$ is nonmonotonous with a maximum at intermediate values and a further decrease to zero.

When the parameter $\delta$ is zero or small(i.e. the Stern layer capacitance is large), a closely packed "diffuse" layer has to form in response to a high potential drop. 


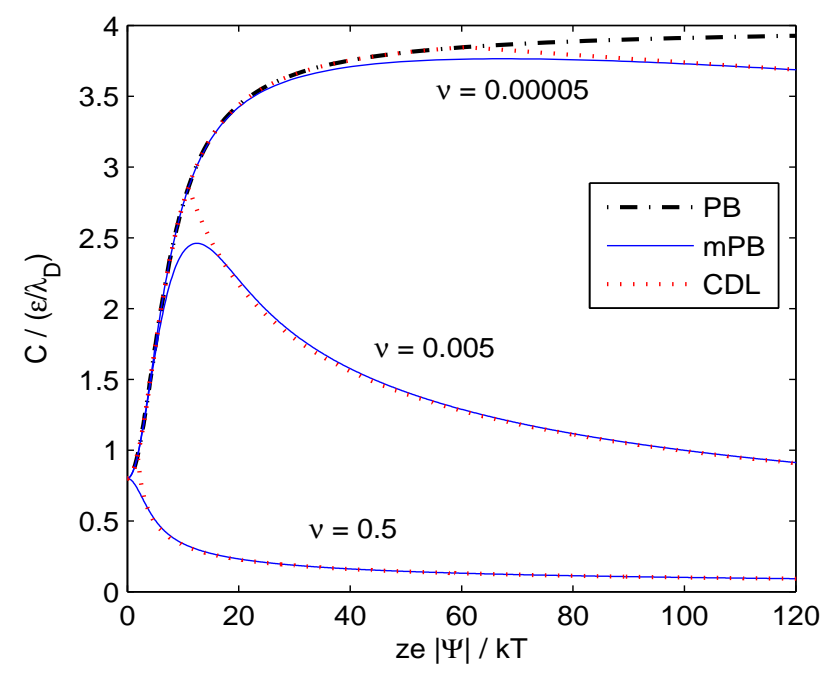

FIG. 10: The total double layer differential capacitance in presence of a Stern layer with $\delta=0.25$ versus the total potential drop across the double layer.

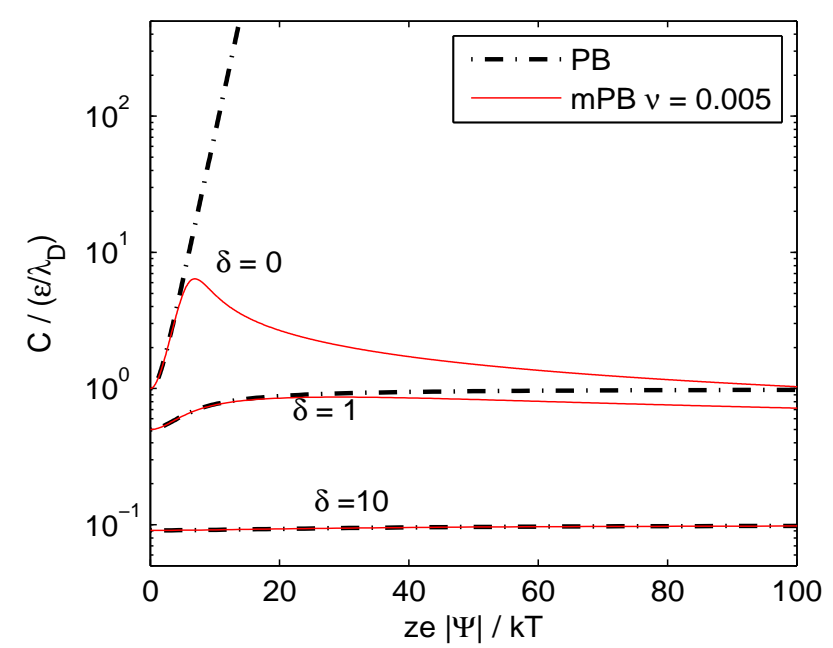

FIG. 11: The differential capacitance $C$ of the double layer as a function of potential difference $\Psi$ for various values of Stern layer capacitance values.

This is the case when the steric effects become important and the PB theory fails. However, for the higher values of $\delta$, most of the potential drop is realized over the Stern layer, and the ions in the diffuse layer are not as densely packed. Thus the system stays below the steric limit, and the PB theory agrees with the MPB theory, as demonstrated by the total capacitance plots of Fig. 11. Similar comments can be made on Fig 10, which shows that for $\delta=0.25$, steric effects may or may not be important depending on value of the dimensionless parameter $\nu=2 a^{3} c_{0}$. For the distribution of the voltage drop over
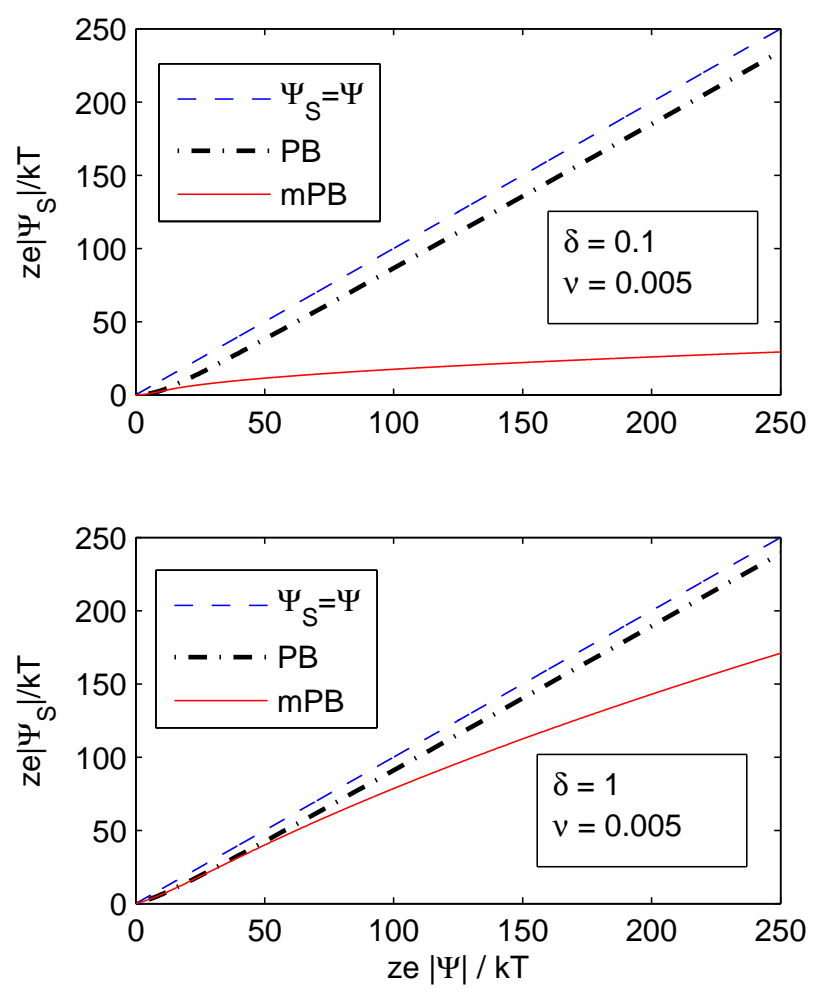

FIG. 12: The Stern layer voltage drop as a function of the total double layer voltage difference. For reference, the line $\Psi_{S}=\Psi$ is also drawn. In the PB theory, almost all of the voltage drop is realized over the Stern layer when the voltage drop is on the order of a few voltages.

the Stern layer and the diffuse layers, see Fig 12

This analysis, although correct, is somewhat misleading, however, since the assumption of a constant compact-layer capacitance may not always be reasonable. In many situations of interest, where several volts $(\approx 100 k T / e)$ are applied across the double layer, it is unlikely that the compact layer could withstand a significant fraction of the total voltage. Note that dielectric breakdown in water can occur in average fields as low as $20 \mathrm{MV} / \mathrm{m}=0.02 \mathrm{~V} / \mathrm{nm}$ in experiments applying submicrosceond voltage pulses (well below the charging time of the double layers) [51]. The critical field may be higher in the Stern layer, where water is confined by ions at the outer Helmholtz plane [13], but it seems implausible for an atomic layer to sustain several volts without somehow "short circuiting" via electron cascades, electrolysis, Faradaic reactions, etc. In some cases, the compact layer models a dielectric thin film, which may be considerably thicker. Again, however, most coating materials, such as teflon or various metal oxides, undergo dielectric breakdown in fields of order $10 \mathrm{MV} / \mathrm{m}$, so a dielectric coating cannot easily withstand several volts unless it is at least $10 \mathrm{~nm}$ wide. 
In general, we see that the capacitance of the compact layer must effectively decrease at large voltages, which corresponds to the limit $\delta \rightarrow 0$. As a result, a signficant fraction of a large voltage must be sustained by the diffuse layer, making steric effects important in many situations of interest. Regardless of the accuracy of our simple models, therefore, we believe that the predicted qualitative effects of ion crowding are likely to have broad relevance for experimental systems applying large voltages.

\section{CONCLUSION}

We have used two simple models for the double layer to account for crowding effects which necessarily take place at intermediate and large applied voltages. These models are both based on modifications of the Poisson Boltzmann description of dilute solutions. This strategy has lead us to identify important operational consequences of these crowding effects the thin double layers, namely a largely reduced double layer capacitance and a decreased ion uptake from the bulk.

We have provided in these sections explicit formulas for the total and differential capacitance and for the salt uptake of interfaces at equilibrium, as a function of the potential drop across the interface. More precisely we have recalled the PB results (no steric effects $\nu=0$ ), and given results for the two simple models with steric effects $(\nu \neq 0)$, considering for all cases the possibility of a finite insulating layer on the electrode $(\delta \neq 0)$.

The two models lead to remarkably similar results suggesting that these semi-quantitatively hold beyond the specifics of these models. Both showed marked differ- ences with the PB approach: the differential capacitance and salt uptake are much weaker, and the former varies non-monotonously with the applied potential.

These observations for the equilibrium properties have led us to make predictions for the dynamics of electrolyte cells of size quite larger than the Debye length: (i) an effective equivalent $\mathrm{RC}$ circuit description holds for a wider range of potentials than expected on the simple basis of the $\mathrm{PB}$ equation, (ii) the response time is much smaller than expected from PB at large voltages, (iii) this time decreases at large voltages after an initial increase for lower values.

The dramatic effect of steric constraints in this problem also shows that other predictions of nonlinear $\mathrm{PB}$ theory, such as the change of scaling from $V^{2}$ to $|V| \log |V|$ for AC electro-osmosis [58], are limited in applicability and should be revisited with models taking crowding effects into account. More generally, this work suggests that, beyond the present problem of ionic transport in electrolytic systems, the description of electrokinetic effects at large applied voltages should be revisited to correct shortcomings of dilute-solution theory.

\section{Acknowledgments}

This work was supported in part by the MRSEC program of the National Science Foundation under award number DMR 02-12383 (MZB). AA acknowledges very gratefully the hospitality of the Department of Mathematics at MIT where this study was realized.
[1] A. Ajdari. AC pumping of liquids. Phys. Rev. E, 61:R45R48, 2000.

[2] S. Alexander, P. M. Chaikin, P. Grant, G. J. Morales, and P. Pincus. Charge renormalization osmotic pressure and bulk modulus of colloidal crystals: theory. J. Chem. Phys., 80:5776-5781, 1984.

[3] M. Z. Bazant and Y. Ben. Theoretical prediction of fast $3 \mathrm{~d}$ ac electro-osmotic pumps. Lab on a Chip, 6:14551461, 2006.

[4] M. Z. Bazant, K. T. Chu, and B. J. Bayly. Currentvoltage relations for electrochemical thin films. SIAM J. Appl. Math., 65:1463-1484, 2005.

[5] M. Z. Bazant and T. M. Squires. Induced-charge electrokinetic phenomena: Theory and microfluidic applications. Phys. Rev. Lett., 92:066101, 2004.

[6] M. Z. Bazant, K. Thornton, and A. Ajdari. Diffuse charge dynamics in electrochemical systems. Phys. Rev. E, 70:021506, 2004.

[7] Martin Z. Bazant and Yuxing Ben. Theoretical prediction of fast 3d ac electro-osmotic pumps. Lab on a Chip, 2006.

[8] L. Belloni. A hypernetted chain study of highly asymmetrical poly-electrolytes. Chem. Phys., 99:43, 1985.

[9] L. Belloni. Ionic condensation and charge renormaliza- tion in colloidal suspensions. Colloids and Surfaces A: Physicochemical and Engineering Aspects, 140:227-243, 1998.

[10] J. J. Bikerman. Z. Phys. Chem. Abt. A, 163:378, 1933.

[11] J. J. Bikerman. Electrokinetic equations and surface conductance. a survey of the diffuse double layer theory of colloidal solutions. Trans. Faraday Soc., 36:154-160, 1940.

[12] Victor A. Bloomfield. Dna condensation by multivalent cations. Biopolymers, 44:269-282, 1998.

[13] J. O.'M Bockris and A. K. N. Reddy. Modern Electrochemistry. Plenum, New York, 1970.

[14] K. Bohinc, A. Iglic, T. Slivnik, and V. Kralj-Iglic. Charged cylindrical surfaces: Effect of finite ion size. Bioelectrochemistry, 57:73-81, 2002.

[15] K. Bohinc, V. Kralj-Iglic, and A. Iglic. Thickness of electrical double layer. effect of ion size. Electrochim. Acta, 46:3033-3040, 2001.

[16] A. Bonnefont, F. Argoul, and M.Z. Bazant. Analysis of diffuse-layer effects on time-dependent interfacial kinetics. J. Electroanal. Chem., 500:52-61, 2001.

[17] I. Borukhov. Charge renormalization of cylinders and spheres: Ion size effects. J. Pol. Sci. B: Pol. Phys., 
42:3598-3620, 2004

[18] I. Borukhov, D. Andelman, and H. Orland. Steric effects in electrolytes: A modified poisson-boltzmann approach. Phys. Rev. Lett., 79:435-438, 1997.

[19] I. Borukhov, D. Andelman, and H. Orland. Adsorption of large ions from an electrolyte solution: A modified poisson boltzmann equation. Electrochim. Acta, 46:221229, 2000.

[20] A. B. D. Brown, C. G. Smith, and A. R. Rennie. Pumping of water with ac electric fields applied to asymmetric pairs of microelectrodes. Phys. Rev. E, 63:016305, 2000.

[21] S. L. Carnie and G. M. Torrie. The statistical mechanics of the electrical double-layer. Adv. Chem. Phys., 46:141$253,1984$.

[22] D. L. Chapman. A contribution to the theory of electrocapillarity. Philos. Mag., 25:475-481, 1913.

[23] K. T. Chu and M. Z. Bazant. Electrochemical thin films at and above the classical limiting current. SIAM J. Appl. Math., 65:1485-1505, 2005.

[24] K. T. Chu and M. Z. Bazant. Nonlinear electrochemical relaxation around conductors. Physical Review E, 74:011501, 2006.

[25] E. Darve D. Saintillan and E. Shaqfeh. Journal of Fluid Mechanics, 2006.

[26] P. Debye and H. Falkenhagen. Physik. Z., 29:121, 1928.

[27] B. V. Deryagin and S. S. Dukhin. The theory of surface conductance. Colloid. J. USSR., 31:277-283, 1969.

[28] N. J. Dudney, J. B. Bates, D. Lubben, and F. X. Hart. Thin-film rechargeable lithium batteries with amorphous $\mathrm{Li}_{x} \mathrm{Mn}_{2} \mathrm{O}_{4}$ cathodes. In J. Bates, editor, Thin Film Solid Ionic Devices and Materials, pages 201-214. The Electrochemical Society, Pennington, NJ, 1995.

[29] S. S. Dukhin. Electrokinetic phenomena of the second kind and their applications. Adv. Colloid Interface Sci., 35:173-196, 1991.

[30] S. S. Dukhin. Non-equilibrium electric surface phenomena. Adv. Colloid Interface Sci., 44:1-134, 1993.

[31] M. Eigen and E. Wicke. The thermodynamics of electrolytes at higher concentration. J. Phys. Chem., 58:702$714,1954$.

[32] V. Freise. Zur theorie der diffusen doppelschicht. Z Elektrochem., 56:822-827, 1952.

[33] J.B. Freund. Electroosmosis in a nano-meter scale channel studied by atomistic simulation. J. Chem. Phys., 116:2194-2200, 2002.

[34] A. Frumkin. Wasserstoffüberspannung und struktur der doppelschict. Z. Phys. Chem., 164A:121-133, 1933.

[35] N. I. Gamayunov, V. A. Murtsovkin, and A. S. Dukhin. Pair interaction of particles in electric field. 1. features of hydrodynamic interaction of polarized particles. Colloid J. USSR, 48:197-203, 1986.

[36] L. A. Geddes. Historical evolution of circuit models for the electrode-electrolyte interface. Ann. Biomedical Eng., 25:1-14, 1997.

[37] D. Gillespie, W. Nonner, and R. S. Eisenberg. Coupling poisson-nernst-planck and density functional theory to calculate ion flux. J. Phys.: Condens. Matter, 14:1212912145, 2002.

[38] D. Gillespie, W. Nonner, and R. S. Eisenberg. Density functional theory of charged, hard-sphere fluids. Phys. Rev. E, 68:031503, 2003.

[39] D. Gillespie, M. Valisko, and D. Boda. Density functional theory of the electrical double layer: the rfd functional. J. Phys.: Condens. Matter, 17:6609-6626, 2005.
[40] A. González, A. Ramos, N. G. Green, A. Castellanos, and H. Morgan. Fluid flow induced by non-uniform ac electric fields in electrolytes on microelectrodes. ii. a linear double-layer analysis. Phys. Rev. E, 61:4019, 2000.

[41] M. Gouy. Sur la constitution de la charge électrique a la surface d'un électrolyte. J. de Phys., 9:457-468, 1910.

[42] L. Guldbrand, B. Jönsson, H. Wennerström, and P. J. Linse. Electrical double-layer forces: A monte carlo study. J. Chem. Phys., 80:2221, 1984.

[43] B.-Y. Ha and Andrea J. Liu. Counterion-mediated attraction between two like-charged rods. Phys. Rev. Lett., 79:1289-1292, 1997.

[44] H. Helmholtz. Studien über electrische grenzschichten. Ann. Phys. Chem., 7 (ser. 3):337-382, 1879.

[45] J. R. Henderson, editor. Fundamentals of Inhomogeneous Fluids. Marcel Dekker, New York, 1992.

[46] M. L. Henle, C. D. Santangelo, D. M. Patel, and P. A. Pincus. Distribution of counterions near discretely charged planes and rods. Europhys. Lett., 66:284-290, 2004.

[47] R. J. Hunter. Foundations of Colloid Science. Oxford University Press, Oxford, 2001.

[48] A. Iglic and V. Kralj-Iglic. Influence of finite size of ions on electrostatic properties of electric double layer. Electrotecnical Rev. (Slovenia), 61:127-133, 1994.

[49] D. Henderson J. Reszko-Zygmunt, S. Sokolowski and D. Boda. Temperature dependence of the double layer capacitance for the restricted primitive model of an electrolyte solution from a density functional approach. $J$. Chem. Phys., 122:084504, 2005.

[50] L. Joly, C. Ybert, E. Trizac, and L. Bocquet. Hydrodynamics within the electric double layer on slipping surfaces. Phys. Rev. Lett., 93:257805, 2004.

[51] H. M. Jones and E. E. Kunhardt. Pulsed dielectric breakdown of pressurized water and salt solutions. Journal of Applied Physics, 77:795-805, 1995.

[52] Bo Jönsson, A. Nonat, C. Labbez, B. Cabane, and H. Wennerström. Controlling the cohesion of cement paste. Langmuir, 21:9211-9221, 2005.

[53] D. Kim and E. Darve. Molecular dynamics simulations of rough wall microchannels. Phys. Rev. E, 73:051203, 2006.

[54] V. Kralj-Iglic and A. Iglic. A simple statistical mechanical approach to the free energy of the electric double layer including the excluded volume effect. J. Phys. II France, 6:477-491, 1996.

[55] C.W. Outhwaite L.B. Bhuiyan and D. Henderson. A modified poisson-boltzmann analysis of the capacitance behaviour of the electric double layer at low temperatures. J. Chem. Phys., 123:034704, 2005.

[56] S. Levine and C.W. Outhwaite. Comparison of theories of the aqueous electric double layer at a charged plane interface. J. Chem. Soc., Faraday Trans. 2, 74:1670, 1977.

[57] J. A. Levitan, S. Devasenathipathy, V. Studer, Y. Ben, T. Thorsen, T. M. Squires, and M. Z. Bazant. Experimental observation of induced-charge electro-osmosis around a metal wire in a microchannel. Colloids and Surfaces A, 267:122-132, 2005.

[58] H. Bruus L.H. Olesen and A. Ajdari. Ac electrokinetic pumps: The effect of geometrical confinement, faradaic current injection and nonlinear surface capacitance. Phys. Rev. E, 73:056313, 2006.

[59] J. Lyklema. Fundamentals of Interface and Colloid Science. Volume I: Fundamentals. Academic Press Limited, 
San Diego, CA, 1991.

[60] J. Lyklema. Fundamentals of Interface and Colloid Science. Volume II: Solid-Liquid Interfaces. Academic Press Limited, San Diego, CA, 1995.

[61] J. Lyklema. Does electrical double layer formation lead to salt exclusion or to uptake? Phys. Rev. E, 71:032501, 2005.

[62] J. R. Macdonald. Theory of the differential capacitance of the double layer in unadsorbed electrolytes. J. Chem. Phys., 22:1857-1866, 1954.

[63] J. R. Macdonald. Impedance spectroscopy: Old problems and new developments. Electrochim. Acta, 35:1483-1492, 1990.

[64] G.S. Manning. Limiting laws and counterion condensation in polyelectrolyte solutions i. colligative properties. J. Chem. Phys., 51:924-933, 1969.

[65] S. Marcelja. Electrostatics of membrane adhesion. Biophys. J., 61:1117, 1992.

[66] V. A. Murtsovkin. Nonlinear flows near polarized disperse particles. Colloid Journal, 58:341-349, 1996.

[67] B. Nadler, Z. Schuss, A. Singer, and R.S. Eisenberg. Diffusion through protein channels: from molecular description to continuum equations. Nanotechnology, 3:439, 2003.

[68] B. Nadler, Z. Schuss, A. Singer, and R.S. Eisenberg. Ionic diffusion through confined geometries: from langevin equations to partial differential equations. J. Phys.: Condens. Matter, 16:S2153-S2165, 2004.

[69] B. J. Neudecker, N. J. Dudney, and J. B. Bates. "lithiumfree" thin-film battery with in situ plated Li anode. J. Electrochem. Soc., 147:517-523, 2000.

[70] J. Newman. Electrochemical Systems. Prentice-Hall, Inc., Englewood Cliffs, NJ, second edition, 1991.

[71] F. Oosawa. Interaction between parallel rodlike macroions. Biopolymers, 6:134, 1968.

[72] F. Oosawa. Polyelectrolytes. Marcel Dekker, New York, 1971.

[73] C.W. Outhwaite and L.B. Bhuiyan. Theory of the electric double layer using a modified poisson-boltzmann equation. J. Chem. Soc., Faraday Trans. 2, 76:1388-1408, 1980.

[74] C.W. Outhwaite and L.B. Bhuiyan. A further treatment of the exclusion-volume term in the modified poissonboltzmann theory of the electric double layer. J. Chem. Soc., Faraday Trans. 2, 78:775-785, 1982.

[75] C.W. Outhwaite and L.B. Bhuiyan. An improved modified poisson-boltzmann equation in electric-double-layer theory. J. Chem. Soc., Faraday Trans. 2, 78:707-718, 1983.

[76] R. Qiao and N. R. Aluru. Ion concentrations and velocity profiles in nanochannel electroosmotic flows. J. Chem. Phys., 118:4692-4701, 2003.

[77] A. Ramos, , H. Morgan, N. G. Green, A. González, and A. Castellanos. Pumping of liquids with traveling-wave electro-osmosis. Journal of Applied Physics, 97:084906, 2005.

[78] A. Ramos, A. González, A. Castellanos, N. G. Green, and H. Morgan. Pumping of liquids with ac voltages applied to asymmetric pairs of microelectrodes. Phys. Rev. E, 67:056302, 2003
79] A. Ramos, H. Morgan, N. G. Green, and A. Castellanos. AC electric-field-induced fluid flow in microelectrodes. $J$. Colloid Interface Sci., 217:420-422, 1999.

[80] R. Roth and D. Gillespie. Physics of size selectivity. Phys. Rev. Lett., 95:247801, 2005.

[81] I. Rubinstein. Electro-Diffusion of Ions. SIAM Studies in Applied Mathematics, SIAM, Philadelphia, PA, 1990.

[82] I. Rubinstein and L. Shtilman. Voltage against current curves of cation exchange membranes. J. Chem. Soc. Faraday. Trans. II, 75:231-246, 1979.

[83] I. Rubinstein and B. Zaltzman. Electro-osmotically induced convection at a permselective membrane. Phys. Rev. E, 62:2238-2251, 2000.

[84] D.J. Mitchell S. L. Carnie, D.Y.C. Chan and B.W. Ninham. The structure of electrolytes at charged surfaces: The primitive model. J. Chem. Phys., 74:1472-1478, 1981.

[85] Christian D. Santangelo. Computing counterion densities at intermediate coupling. cond-mat/0501498.

[86] Z. Schuss, B. Nadler, and R.S. Eisenberg. Derivation of pnp equations in bath and channel from a molecular model. Physical Review E, 64:036116, 2001.

[87] V. N. Shilov and T. S. Simonova. Polarization of electric double layer of disperse particles and dipolophoresis in a steady (dc) field. Colloid J. USSR, 43:90-96, 1981.

[88] I. N. Simonov and V. N. Shilov. Theory of low-frequency dielectric-dispersion of a suspension of ideally polarizable spherical particles. Colloid J. USSR, 39:775-780, 1977.

[89] T. A. Simonova, V. N. Shilov, and O. A. Shramko. Low-frequency dielectrophoresis and teh polarization interaction of uncharged spherical particles with an induced deby atmosphere of arbitrary thickness. Colloid J., 63:108-115, 2001.

[90] M. Sluyters-Rehbach and J. H. Sluyters. Electroanalytical Chemistry, volume 4, pages 1-128. Marcel Dekker, New York, 1970.

[91] W. H. Smyrl and J. Newman. Double layer structure at the limiting current. Trans. Faraday Soc., pages 207-216, 1967.

[92] T. M. Squires and M. Z. Bazant. Induced-charge electroosmosis. J. Fluid Mech., 509:217-252, 2004.

[93] T. M. Squires and M. Z. Bazant. Breaking symmetries in induced-charge electro-osmosis and electrophoresis. $J$. Fluid Mech., 560:65-101, 2006.

[94] O. Stern. Zur theorie der electrolytischen doppelschicht. Z. Elektrochem., 30:508-516, 1924.

[95] N. Takami, T. Ohsaki, H. Hasabe, and M. Yamamoto. Laminated thin Li-ion batteries using a liquid electrolyte. J. Electrochem. Soc., 148:A9-A12, 2002.

[96] B. Wang, J. B. Bates, F. X. Hart, B. C. Sales, R. A. Zuhr, and J. D. Robertson. Characterization of thin-film rechargeable lithium batteries with lithium cobalt oxide cathodes. J. Electrochem. Soc., 143:3204-3213, 1996.

[97] E. Wicke and M. Eigen. ber den einflu des raumbedarfs von ionen in wssriger lsung auf ihre verteilung im elektrischen feld und ihre aktivittskoeffizienten. Z. Elektrochem., 56:551, 1952.

[98] E. Yariv. Induced-charge electrophoresis of nonspherical particles. Phys. Fluids, 17:051702, 2005. 